\title{
UNFOLDING FINITIST ARITHMETIC
}

\author{
SOLOMON FEFERMAN \\ Stanford University \\ and \\ THOMAS STRAHM \\ University of Bern
}

\begin{abstract}
The concept of the (full) unfolding $\mathcal{U}(\mathrm{S})$ of a schematic system $\mathrm{S}$ is used to answer the following question: Which operations and predicates, and which principles concerning them, ought to be accepted if one has accepted $\mathrm{S}$ ? The program to determine $\mathcal{U}(\mathrm{S})$ for various systems $\mathrm{S}$ of foundational significance was previously carried out for a system of nonfinitist arithmetic, NFA; it was shown that $\mathcal{U}$ (NFA) is proof-theoretically equivalent to predicative analysis. In the present paper we work out the unfolding notions for a basic schematic system of finitist arithmetic, FA, and for an extension of that by a form $\mathrm{BR}$ of the so-called Bar Rule. It is shown that $\mathcal{U}(\mathrm{FA})$ and $\mathcal{U}$ (FA+BR) are proof-theoretically equivalent, respectively, to Primitive Recursive Arithmetic, PRA, and to Peano Arithmetic, PA.
\end{abstract}

§1. Introduction. This is a continuation of the program introduced in Feferman (1996), to determine the unfolding of the principal foundational schematic systems $\mathrm{S}$, from arithmetic through analysis up to set theory. Roughly speaking, the concept of the full unfolding of $\mathrm{S}, \mathcal{U}(\mathrm{S})$, is used to answer the following question:

Given a schematic system S, which operations and predicates, and which principles concerning them, ought to be accepted if one has accepted $\mathrm{S} ?^{1}$

A quite general theory of operations and predicates serves as the framework in which to formulate this notion. Then, for any specific $S$ we expand that to include the basic operations given on the universe of discourse of $S$ together with the basic logical operations used to construct the predicates of S.

The preceding article in this series, Feferman \& Strahm (2000), provided the first example of these notions worked out in detail, namely for a schematic system of classical nonfinitist arithmetic, NFA. Its basic operations on individuals with the constant 0 are successor, Sc, and predecessor, $\mathrm{Pd}$; the basic logical operations are $\neg, \wedge$, and $\forall$. It is given by the following axioms, where we write as usual, $x^{\prime}$ for $\operatorname{Sc}(x)$ :

(1) $x^{\prime} \neq 0$

Received: November 26, 2009

1 Actually, three levels of unfolding were proposed in Feferman (1996): they are, in increasing order, $\mathcal{U}_{0}(\mathrm{~S}), \mathcal{U}_{1}(\mathrm{~S})$, and $\mathcal{U}(\mathrm{S}) . \mathcal{U}_{0}(\mathrm{~S})$ is the operational unfolding of $\mathrm{S}$, that is, it only concerns the operational part of our basic question; the full unfolding of $S, \mathcal{U}(\mathrm{S})$, adds to $\mathcal{U}_{0}(\mathrm{~S})$ the predicates that ought to be accepted if one has accepted $S$, including those generated by a kind of join operation; the intermediate system $\mathcal{U}_{1}(\mathrm{~S})$ is like $\mathcal{U}(\mathrm{S})$ without use of that operation. 
(2) $\operatorname{Pd}\left(x^{\prime}\right)=x$

(3) $P(0) \wedge(\forall x)\left[P(x) \rightarrow P\left(x^{\prime}\right)\right] \rightarrow(\forall x) P(x)$.

Here $P$ is a free predicate variable, and the intention is to use the induction scheme (3) in a wider sense than is limited by the basic language of NFA or any language fixed in advance. Namely, one applies the general rule of substitution

$$
A[P] \Rightarrow A[B / P]
$$

to any formulas $A$ and $B$ that arise in the process of unfolding NFA. The means for carrying out that process is provided by our background theory of operations, which includes a general scheme of recursive definition of partial operations, to which induction may be applied successively to verify that more and more operations are defined for all values, and similarly for predicates. The main result of Feferman \& Strahm (2000) is that $\mathcal{U}(\mathrm{NFA})$ is proof-theoretically equivalent to predicative analysis. ${ }^{2}$ In Section 2 below we review the work on $\mathcal{U}$ (NFA) in more detail both as a preliminary to the new work here on finitist arithmetic, as well as to show how it may be simplified by use of a background theory of operations in the partial combinatory calculus.

In the present paper we work out the unfolding notions first for a system of finitist arithmetic, FA, and then for an extension of that by a form BR of the so-called Bar Rule. These are both provided with the same basic operations on individuals as given for NFA. But the logical operations now are restricted to $\wedge, \vee$, and $\exists$. Provable propositions $A(x)$ are interpreted as verifying $A(n)$ for each natural number $n$, but we do not have universal quantification over the natural numbers as a logical operation. Nor do we have negation (except of numerical equations) which when applied to formulas of the form $(\exists x) A(x)$ could be interpreted as having the effect of universal quantification. It will be shown that $\mathcal{U}(\mathrm{FA})$ contains the quantifier-free system of Primitive Recursive Arithmetic, PRA. In the verification of this we reason about recursion equations of the form

$$
F(x, y)=G(x) \text { if } y=0 \text {, else } H(x, \operatorname{Pd}(y), F(\operatorname{Pd}(y))) .
$$

If $G, H$ have already been recognized to be total operations, then we prove that $F$ is total-that is, that $F(x, y)$ is defined for each $y$-by induction on $y$. This makes use of our framework notion of a term $t$ being defined -in symbols, $t \downarrow$ - which is a special kind of existential statement. In order to reason from such statements to new such statements given the above restriction of the logical operations of FA, we make use of a sequent formulation of our calculus, that is, the statements proved are sequents $\Sigma$ of the form $\Gamma \rightarrow A$, where $\Gamma$ is a finite sequence (possibly empty) of formulas, and $A$ may also be the false proposition $\perp$. Moreover, induction must now be given as a rule of inference involving such sequents. In these terms, the basic axioms and rules of FA are as follows:

(1) $x^{\prime}=0 \rightarrow \perp$

(2) $\operatorname{Pd}\left(x^{\prime}\right)=x$

(3) $\frac{\Gamma \rightarrow P(0) \quad \Gamma, P(x) \rightarrow P\left(x^{\prime}\right)}{\Gamma \rightarrow P(x)}$.

2 In more detail, the results of Feferman \& Strahm (2000) are that $\mathcal{U}_{0}(\mathrm{NFA}) \equiv \mathrm{PA}, \mathcal{U}_{1}(\mathrm{NFA}) \equiv$ $\mathrm{RA}_{<\omega}$, and $\mathcal{U}(\mathrm{NFA}) \equiv \mathrm{RA}_{<\Gamma_{0}}$, where, as usual, PA is the system of Peano Arithmetic, $\mathrm{RA}<\alpha$ denotes the system of ramified analysis in levels $<\alpha, \Gamma_{0}$ is the so-called Feferman-Schütte ordinal that measures the limit of predicative reasoning, and the relation $\equiv$ is that of prooftheoretical equivalence, with conservation from left to right for suitable classes of formulas. 
Now the appropriate substitution rule (Subst') takes us from any inferred rule of inference $\Sigma_{1}(P), \ldots, \Sigma_{n}(P) \Rightarrow \Sigma(P)$, to the result of substituting a formula $B$ for $P$ throughout. The main result obtained for the unfolding of this system in Section 3 below is that $\mathcal{U}(\mathrm{FA}) \equiv$ PRA. $^{3}$

The formulation of a version BR of the Bar Rule under the above formal restrictions is introduced in Section 4, where it is shown how nested recursion on an ordering for which the no descending sequences property NDS has been verified can be inferred from a suitable form of induction on that ordering. ${ }^{4}$ It follows from the work of Tait (1961), that the NDS property can be verified in $\mathcal{U}_{0}(\mathrm{FA}+\mathrm{BR})$ for the natural ordering associated with each ordinal less than Cantor's ordinal $\varepsilon_{0}$. (A compact form of the argument for that, communicated to us by Tait, is presented in the Appendix to this article.) This shows that $\mathrm{PA}$ is a lower bound to the proof-theoretical strength of $\mathcal{U}_{0}(\mathrm{FA}+\mathrm{BR})$. The work on the unfolding of this system is completed with the proof that the full unfolding $\mathcal{U}(\mathrm{FA}+\mathrm{BR})$ does not go beyond PA in strength.

Before going into the detailed work, something must be said in the remainder of this introduction about how our formulation of FA and its possible extension by BR relates to the extensive literature on finitism, both informal and formal, which has its source in Hilbert's consistency program. This must necessarily be comparatively brief and we shall just cite a few references; the online encyclopedia article Zach (2003) provides an excellent introduction and many further key references; see also Zach (2006) for an expanded version of Zach (2003).

Hilbert viewed reasoning about the actual infinite as the source of possible inconsistencies in mathematics. He thus proposed to establish the consistency of stronger and stronger formal systems for mathematics, beginning with that for Peano Arithmetic, by means entirely of finitist reasoning from which all references to the actual infinite, explicit or implicit, would have to be excluded. Following some suggestions by Hilbert as to how his program might be carried out, initial contributions to it were made by Bernays, Ackermann, von Neumann, and Herbrand; however, none went beyond weak subsystems of PA. Whether there is any limit to what could be accomplished by purely finitary means would have to depend on a precise explanation of what are the allowed objects and methods of proof of finitism. But Hilbert was rather vague about both of these, saying such things as that it relies entirely on a "purely intuitive basis of concrete signs." These signs are finite sequences of symbols, for example, as given by the expressions of a formal system, of which the most basic such signs are the tallies $||,||||,, \ldots$ representing the positive integers. ${ }^{5}$ Given that idea of its subject matter, what are the allowed finitistic methods of definition and proof? Even in the great collaboration with Bernays, Grundlagen der Mathematik Hilbert \& Bernays (1968, 1970), there is no detailed explanation of that. Given Hilbert's great optimism about the prospects for his program without limit it may be that he thought people would recognize any piece of reasoning used to carry it out as finitist on the face of it, without requiring any general explanation of what makes it so. At any rate, one gleans from Hilbert \& Bernays (1968), pp. 32ff that finitism at least includes

3 In this case, all three unfoldings of FA have the same strength; that result was announced in Feferman \& Strahm (2001).

4 NDS is formulated via the adjunction of a free ("anonymous") function constant $f$ for a possible infinite descending sequence.

5 As Gödel showed by his arithmetization of syntax, the former can be reduced to the latter. 
PRA. ${ }^{6}$ It is a matter of some historical discussion whether Hilbert accepted as finitist certain operations and inferences going beyond PRA; the evidence according to Zach (2006), p. 425, is that he did, at least implicitly, since he accepted the proof (by Ackermann, among others) of consistency of a system corresponding to PRA necessarily making use of stronger principles.

Gödel's (1931) second incompleteness theorem led von Neumann to the conclusion that Hilbert's program could not succeed for PA; Gödel thought at first that it might, but within a few years he came around to the same opinion. In order for that to be definitive, the crucial question would depend on a precise explanation of how finitism ought to be characterized, independently of the historical question of what Hilbert and his circle judged particular arguments to be, or not be, finitistic. Gödel's own thoughts on this will be described below. The first proposed formal characterization was made by Kreisel (1960), then in a revised form in his article Kreisel (1965), with further discussion in Kreisel (1970); according to that, finitism is equivalent in strength to PA. The second proposed formal characterization was made by Tait in Tait (1968) and Tait (1981), the latter reprinted in Tait (2005); according to that, finitism is equivalent in strength to PRA. We shall take up these formulations in reverse order. Both agree that it makes sense to characterize the objects and methods of finitism only from a nonfinitist point of view. ${ }^{7}$

On Tait's view, the essence of finitism lies in the rejection of all reference to infinite totalities. In particular functions on the natural numbers qua sets of order pairs cannot be part of the subject matter of finitism, not even finitist functions in general. To verify that a particular function $F$ on the natural numbers given by a certain rule is finitist one must have a finitist proof that shows how to construct for each possible argument its value under $F$. Suppose, for example, $G$ and $H$ are given finitist functions, and $F$ is introduced by the equations $F(x, 0)=G(x)$, and $F\left(x, y^{\prime}\right)=H(x, y, F(x, y))$. The argument that $F$ is finitist comes from the recognition that the construction of $F(x, y)$ for $y \neq 0$ is reduced to that of $F(x, \operatorname{Pd}(y))$ and that the sequence $y, \operatorname{Pd}(y), \operatorname{Pd}(\operatorname{Pd}(y))$ terminates with 0 . The finitist functions are evidently also closed under the other procedures generating the primitive recursive functions, so by this argument each primitive recursive function is finitist. This led Tait to state the following:

THESIS. The finitist functions are precisely the primitive recursive functions (Tait, 2005, p. 29).

Concerning the upper bound here, Tait says that one can't prove that the finitist functions do not go beyond those that are primitive recursive, since the concept of finitist function is not a rigorous one (the situation is analogous to that of Church's Thesis). Rather, "[w]e must argue that every plausible attempt to construct a finitist function that is not primitive recursive either fails to be finitist according to our specifications or turns out to be primitive recursive after all" (Tait, 2005). An ancillary argument is made for the thesis that the finitist proofs are just those that can be formalized in PRA.

6 Parsons (1998) has argued that the ideas of concrete intuition expressed by Hilbert do not allow one to go beyond what can be obtained by addition, multiplication, and bounded quantification; if that is granted, not even exponentiation would be accepted as a finitist operation.

7 This is of course analogous to the argument that a characterization of predicative definability and provability can only be given from an impredicative standpoint. 
As presented in Kreisel (1965), pp. 169-172, Kreisel's characterization of finitist proof is given in terms of an autonomous progression of quantifier free systems, $T_{\alpha}$, employing an auxiliary predicate $O(\xi)$ interpreted as expressing that the (concrete) structure showing how the ordinal $\xi$ is built up can be finitistically visualized. It is assumed that if we have derived $O(\alpha)$ then we can infer $O(\alpha \omega)$, that is, we can visualize an $\omega$-sequence of copies of $\alpha$; furthermore, we can infer iteration of a previously recognized operation $\alpha$ times. The essential autonomy condition is that one can proceed to stage $T_{\alpha}$ if one has a proof in an earlier $T_{\beta}$ that all $\xi<\alpha$ can be visualized. The result that the autonomous ordinals are exactly those less than $\varepsilon_{0}$ is stated without proof in Kreisel (1965), p. 172; it follows that the union of the autonomous systems $T_{\alpha}$ is proof-theoretically equivalent to PA.

Let us return to Gödel's views on the limits of finitism. This has been discussed at length in Feferman (to appear), where much of the evidence rests on his posthumously published notes for a 1933 lecture in Cambridge, Massachusetts Gödel (1995) and a 1938 lecture to Zilsel's seminar in Vienna Gödel (1995), as well as on extended correspondence with Bernays, reproduced in Gödel (2003). In both the 1933 and 1938 lectures Gödel informally describes several levels of constructivity, and equates finitist reasoning with the lowest level, given by means of a system $A$ that is to meet several conditions. The system $A$ has been interpreted by its commentators as a form of PRA. Among the conditions on A, though, is one that suggests that existential quantification may be formally employed in positive contexts:

Negatives of general propositions (i.e. existence propositions) are to have a meaning in our system only in the sense that we have found an example but, for the sake of brevity, do not wish to state it explicitly. I.e., they serve merely as an abbreviation and could be entirely dispensed with if we wished. (Gödel 1933, p. 51).

Thus, among these proposed characterizations, our own formulation of FA may be considered to be closest to that of Gödel. On the other hand, that of FA + BR is closest to a suggestion made by Kreisel "for a more attractive formulation" in Kreisel (1965), p. 173 directly following his proposed characterization in terms of autonomous progressions. Namely, that is to add to PRA "free function variables and a constructive existential numerical quantifier with the obvious rules" plus the inference rule from NDS on a given ordering $R$ to a suitable form of transfinite induction on $R$ for existential formulas. He states that it is sufficient to infer nested recursion on $R$ and thence to use Tait (1961) to justify induction on each ordinal less than $\varepsilon_{0} .{ }^{8}$ Interestingly, though Gödel and Kreisel were in close contact in the 1960s and Gödel was well aware of the latter's proposed characterization of finitism via an autonomous progression, he did not refer to this suggestion in his letter of 25 July 1969 to Bernays in which he toyed with the idea that a suitable formulation of BR that brings one up to $\varepsilon_{0}$ comes close to finitism (cf., Gödel, 2003, p. 271).

Our aim here is not to argue for any one principled view of how finitism ought to be characterized. Rather, our purpose is to point out that there are natural formulations in terms of schematic systems for which the unfolding process yields in one case a system

8 However, Kreisel's particular statement in (1965), p. 173 of the form of transfinite induction on $R$ for existential formulas is prima facie logically defective and not at all adequate to its intended purpose. 
equivalent to PRA and in the other case a system equivalent to PA. We hope that this way of looking at finitism may be useful to provide grounds for further discussion on which to bolster or reject one or the other characterizations previously on offer. Aside from that, we believe the apparatus of FA and perhaps some or all of its extension by BR comes closer to reflecting the actual practice of finitism than the systems previously considered.

\$2. The unfolding of NFA revisited. The aim of this section is twofold. First, we want to set up a modified version of unfolding which leads to a simplification of the unfolding systems presented in Feferman (1996) and Feferman \& Strahm (2000). Moreover, using this new unfolding notion, we will restate the results obtained in Feferman \& Strahm (2000) concerning the proof-theoretic strength of the unfolding of the basic schematic system NFA of nonfinitist arithmetic.

To begin with we will describe the unfolding of a schematic system $S$ informally by stating some general methodological "pre-axioms." Then we will spell out these axioms in all detail for S being the schematic system NFA.

Underlying the idea of unfolding for arbitrary $S$ are general notions of (partial) operation and predicate, belonging to a universe $V$ encompassing the universe of discourse of $\mathrm{S}$. Both are considered to be intensional entities, given by rules of computation and defining properties, respectively. Operations are not bound to any specific mathematical domain, but have to be considered as premathematical in nature. Operations can apply to other operations as well as to predicates. Some operations are universal and are naturally selfapplicable as a result, like the identity operation or the pairing operation, while some are partial and presented to us on specific mathematical domains only, like addition on the natural numbers or the real numbers. Operations satisfy the laws of a partial combinatory algebra with pairing, projections, and definition by cases. Predicates are equipped with a membership relation $\in$ to express that given elements satisfy the predicate's defining property.

For the formulation of the full unfolding $\mathcal{U}(\mathrm{S})$ of any given schematic axiom system $\mathrm{S}$, we have the following axioms.

1. The universe of discourse of $\mathrm{S}$ has associated with it an additional unary relation symbol, $U_{S}$, and the axioms of $S$ are relativized to $U_{S}$. (Similarly if $S$ is manysorted).

2. Each $n$-ary operation symbol $f$ of $\mathbf{S}$ determines an element $f^{\star}$ of our partial combinatory algebra, with $f\left(x_{1}, \ldots, x_{n}\right)=f^{\star} x_{1} \ldots x_{n}$ on $\mathrm{U}_{\mathrm{S}}^{n}$ (or the domain of $f$ in case $f$ itself is given as a partial operation).

3. Each relation symbol $R$ of $\mathrm{S}$ together with $\mathrm{U}_{\mathrm{S}}$ determines a predicate $R^{\star}$ with $R\left(x_{1}, \ldots, x_{n}\right)$ if and only if $\left(x_{1}, \ldots, x_{n}\right) \in R^{\star}$.

4. Operations on predicates, such as, for example, conjunction, are just special kinds of operations. Each logical operation $l$ of $\mathrm{S}$ determines a corresponding operation $l^{\star}$ on predicates.

5. Sequences of predicates given by an operation $f$ form a new predicate $\operatorname{Join}(f)$, the disjoint union of the predicates from $f$.

Moreover, the free predicate variables $P, Q, \ldots$ used in the schematic formulation of $\mathrm{S}$ give rise to the crucial rule of substitution (Subst), according to which we are allowed to 
substitute any formula $B$ for $P$ in a previously recognized (i.e., derived) statement $A[P]$ depending on $P$.

The restriction $\mathcal{U}_{0}(\mathrm{~S})$ of $\mathcal{U}(\mathrm{S})$ is obtained by dropping the axioms concerning predicates; $\mathcal{U}_{0}(\mathrm{~S})$ is called the operational unfolding of $\mathrm{S}$. Moreover, there is a natural intermediate predicate unfolding system $\mathcal{U}_{1}(\mathrm{~S})$, which is simply $\mathcal{U}(\mathrm{S})$ without the predicate forming operation of Join.

The following spells out in detail the three unfolding systems $\mathcal{U}_{0}(\mathrm{~S}), \mathcal{U}_{1}(\mathrm{~S})$, and $\mathcal{U}(\mathrm{S})$ for $S=N F A$, the schematic system of nonfinitist arithmetic. Recall that the specified basic logical operations of NFA are $\neg, \wedge$, and $\forall$. Its axioms simply include the usual ones for 0 , $\mathrm{Sc}$ and $\mathrm{Pd}$, as well as induction stated in its standard schematic form using a free predicate variable $P$,

$$
P(0) \wedge(\forall x)\left(P(x) \rightarrow P\left(x^{\prime}\right)\right) \rightarrow(\forall x) P(x) .
$$

We begin with the operational unfolding $\mathcal{U}_{0}$ (NFA). Its language is first order, using variables $a, b, c, f, g, h, u, v, w, x, y, z \ldots$ (possibly with subscripts). It includes (i) the constant 0 and the unary function symbols Sc and Pd of NFA, (ii) constants for operations as individuals, namely sc, $\mathrm{pd}$ (successor, predecessor), $\mathrm{k}, \mathrm{s}$ (combinators), $\mathrm{p}, \mathrm{p}_{0}, \mathrm{p}_{1}$ (pairing and unpairing), d, t, ff (definition by cases, true, false), and e (equality), and (iii) a binary function symbol · for (partial) term application. Further, we have (iv) a unary relation symbol $\downarrow$ (defined) and a binary relation symbol $=$ (equality), as well as (v) a unary relation symbol $\mathrm{N}$ (natural numbers). In addition, we have a symbol $\perp$ for the false proposition. Finally, a stock of free predicate symbols $P, Q, R, \ldots$ of finite arities is assumed. ${ }^{9}$

The terms $(r, s, t, \ldots)$ of $\mathcal{U}_{0}$ (NFA) are inductively generated from the variables and constants by means of the function symbols $\mathrm{Sc}, \mathrm{Pd}$, as well as · for application. In the following we often abbreviate $(s \cdot t)$ simply as $(s t)$, st or sometimes also $s(t)$; the context will always ensure that no confusion arises. We further adopt the convention of association to the left so that $s_{1} s_{2} \ldots s_{n}$ stands for $\left(\ldots\left(s_{1} s_{2}\right) \ldots s_{n}\right)$. Further, we put $t^{\prime}:=\operatorname{Sc}(t)$ and $1:=0^{\prime}$. We define general $n$-tupling by induction on $n \geq 2$ as follows:

$$
\left(s_{1}, s_{2}\right):=\mathrm{p}_{1} s_{2}, \quad\left(s_{1}, \ldots, s_{n+1}\right):=\left(\left(s_{1}, \ldots, s_{n}\right), s_{n+1}\right) .
$$

Moreover, we set $(s):=s$ and ()$:=0$.

The formulas $(A, B, C, \ldots)$ of $\mathcal{U}_{0}(\mathrm{NFA})$ are inductively generated from the atomic formulas $\perp, s \downarrow,(s=t), \mathrm{N}(s)$, and $P\left(s_{1}, \ldots, s_{n}\right)$ by means of negation $\neg$, conjunction $\wedge$, and universal quantification $\forall$. The remaining logical connectives and quantifiers are defined as usual by making use of classical logic.

The sequence notation $\bar{u}$ and $\bar{t}$ is used in order to denote finite sequences of variables and terms, respectively. Moreover, we write $t[\bar{u}]$ to indicate a sequence $\bar{u}$ of free variables possibly appearing in the term $t$; however, $t$ may contain other variables than those shown by using this bracket notation. Further, $t[\bar{s}]$ is used to denote the result of simultaneous substitution of the terms $\bar{s}$ for the variables $\bar{u}$ in the term $t[\bar{u}]$. The meaning of $A[\bar{u}]$ and $A[\bar{s}]$ is understood accordingly. Finally, we will also use the sequence notation $\bar{A}$ in order to denote a finite sequence $\bar{A}=A_{1}, \ldots, A_{n}$ of formulas.

$\mathcal{U}_{0}$ (NFA) is based on partial term application. Hence, it is not guaranteed that terms have a value, and $t \downarrow$ is read as " $t$ is defined" or " $t$ has a value". Accordingly, the partial

9 The constants Sc and pd as well as the relation symbol $\mathrm{N}$ are used instead of the symbols $\mathrm{Sc}^{\star}$, $\mathrm{Pd}^{\star}$, and $\mathrm{U}_{\mathrm{NFA}}$ mentioned in the informal description above. 
equality relation $\simeq$ is introduced by

$$
s \simeq t:=(s \downarrow \vee t \downarrow) \rightarrow(s=t) .
$$

Further, we will use the following abbreviations concerning the predicate $\mathrm{N}$ for the natural numbers $\left(\bar{s}=s_{1}, \ldots, s_{n}\right)$ :

$$
\begin{aligned}
\bar{s} \in \mathrm{N} & :=\mathrm{N}\left(s_{1}\right) \wedge \cdots \wedge \mathrm{N}\left(s_{n}\right), \\
(\exists x \in \mathrm{N}) A & :=(\exists x)(x \in \mathrm{N} \wedge A), \\
(\forall x \in \mathrm{N}) A & :=(\forall x)(x \in \mathrm{N} \rightarrow A) .
\end{aligned}
$$

The logic of $\mathcal{U}_{0}(\mathrm{NFA})$ is the classical logic of partial terms LPT of Beeson (1985), cf., also Feferman (1975). We recall that LPT embodies strictness axioms saying that all subterms of a defined compound term are defined as well. Moreover, if $(s=t)$ holds then both $s$ and $t$ are defined, and $s$ is defined provided $\mathrm{N}(s)$ holds, and similarly for $P(\bar{s})$.

The axioms of $\mathcal{U}_{0}$ (NFA) are divided into three groups as follows.

\section{Embedding of NFA}

(1) The relativization of the axioms of NFA to the predicate $\mathrm{N},{ }^{10}$

(2) $(\forall x \in \mathrm{N})[\operatorname{Sc}(x)=\operatorname{sc}(x) \wedge \operatorname{Pd}(x)=\operatorname{pd}(x)]$.

II. Partial combinatory algebra, pairing, definition by cases

(3) $\mathrm{k} a b=a$,

(4) $\mathrm{s} a b \downarrow \wedge \mathrm{s} a b c \simeq a c(b c)$,

(5) $\mathrm{p}_{0}(a, b)=a \wedge \mathrm{p}_{1}(a, b)=b$,

(6) $\mathrm{d} a b \mathbf{t}=a \wedge \mathrm{d} a b \boldsymbol{f}=b$.

\section{Equality on the natural numbers $\mathrm{N}$}

(7) $(\forall x, y \in \mathrm{N})[\mathrm{e} x y=\mathbb{t} \vee \mathrm{e} x y=\mathrm{ff}]$,

(8) $(\forall x, y \in \mathrm{N})[\mathrm{e} x y=\mathbb{t} \leftrightarrow x=y]$.

The axioms in Group I describe the standard embedding of NFA into its operational unfolding system $\mathcal{U}_{0}$ (NFA). In particular, the unary functions $\mathrm{Sc}$ and $\mathrm{Pd}$ are represented by corresponding operations SC and pd. The heart of the axioms in Group II are the laws concerning the combinators $\mathrm{k}$ and $\mathrm{s}$. As usual, the axioms of a partial combinatory algebra allow one to define $\lambda$-abstraction and to prove a recursion or fixed point theorem for applicative terms. For proofs of these standard results the reader is referred to Beeson (1985); Feferman (1975).

Whereas the axioms in Group II are basic for the unfolding of any schematic system $S$, the axioms in Group III about the decidability of equality on the natural numbers are specific for the unfolding system of NFA. For other systems S, one may leave it open which equality functions are accepted as basic.

Finally, crucial for the formulation of $\mathcal{U}_{0}(\mathrm{~S})$ is the predicate substitution rule:

$$
A[\bar{P}] \Rightarrow A[\bar{B} / \bar{P}] \text {. }
$$

10 Note that this relativization also includes axioms such as $0 \in \mathrm{N}$ and $(\forall x \in \mathrm{N})\left(x^{\prime} \in \mathrm{N}\right)$. 
Here $\bar{P}=P_{1}, \ldots, P_{m}$ is a sequence of free predicate symbols possibly appearing in the formula $A[\bar{P}]$ and $\bar{B}=B_{1}, \ldots, B_{m}$ is a sequence of formulas. In the conclusion of this rule of inference, $A[\bar{B} / \bar{P}]$ denotes the formula $A[\bar{P}]$ with each subformula $P_{i}(\bar{t})$ replaced by $(\exists \bar{x})\left(\bar{t}=\bar{x} \wedge B_{i}[\bar{x}]\right)$, where the length of $\bar{x}$ equals the arity of $P_{i}$.

We now turn to the full predicate unfolding $\mathcal{U}(\mathrm{NFA})$ and its restriction $\mathcal{U}_{1}$ (NFA).

The language of $\mathcal{U}(\mathrm{NFA})$ extends the language of $\mathcal{U}_{0}$ (NFA) by additional constants nat (natural numbers), eq (equality), $\operatorname{pr}_{P}$ (free predicate $P$ ), inv (inverse image), neg (negation), conj (conjunction), un (universal quantification), and join (disjoint unions). In addition, we have a new unary relation symbol $\Pi$ for (codes of) predicates and a binary relation symbol $\in$ for expressing elementhood between individuals and predicates, that is, satisfaction of those predicates by the given individuals. The terms of $\mathcal{U}$ (NFA) are generated as before but now taking into account the new constants. The formulas of $\mathcal{U}(\mathrm{NFA})$ extend the formulas of $\mathcal{U}_{0}$ (NFA) by allowing new atomic formulas of the form $\Pi(t)$ and $s \in t$.

The axioms of $\mathcal{U}(\mathrm{NFA})$ extend those of $\mathcal{U}_{0}(\mathrm{NFA})$, as follows.

\section{Basic axioms about predicates}

(9) $\Pi$ (nat) $\wedge(\forall x)(x \in$ nat $\leftrightarrow \mathrm{N}(x)),{ }^{11}$

(10) $\Pi(\mathrm{eq}) \wedge(\forall x)(x \in \mathrm{eq} \leftrightarrow(\exists y)(x=(y, y)))$,

(11) $\Pi\left(\operatorname{pr}_{P}\right) \wedge(\forall \bar{x})\left((\bar{x}) \in \operatorname{pr}_{P} \leftrightarrow P(\bar{x})\right)$,

(12) $\Pi(a) \rightarrow \Pi(\operatorname{inv}(a, f)) \wedge(\forall x)(x \in \operatorname{inv}(a, f) \leftrightarrow f x \in a)$,

(13) $\Pi(a) \rightarrow \Pi(\operatorname{neg}(a)) \wedge(\forall x)(x \in \operatorname{neg}(a) \leftrightarrow x \notin a)$,

(14) $\Pi(a) \wedge \Pi(b) \rightarrow \Pi(\operatorname{conj}(a, b)) \wedge(\forall x)(x \in \operatorname{conj}(a, b) \leftrightarrow x \in a \wedge x \in b)$,

(15) $\Pi(a) \rightarrow \Pi(\mathrm{un}(a)) \wedge(\forall x)(x \in \operatorname{un}(a) \leftrightarrow(\forall y \in \mathrm{N})((x, y) \in a))$.

\section{Join axiom}

(16) $(\forall x \in \mathrm{N}) \Pi(f x) \rightarrow \Pi($ join $(f)) \wedge(\forall x)(x \in \operatorname{join}(f) \leftrightarrow J[f, x])$,

where $J[f, u]$ expresses that $u$ is an element of the disjoint union of $f$ over $\mathbf{N}$, that is,

$$
J[f, u]:=(\exists y \in \mathrm{N})(\exists z)(u=(y, z) \wedge z \in f y) .
$$

In addition, $\mathcal{U}(\mathrm{NFA})$ contains the substitution rule (Subst), that is, the rule $A[\bar{P}] \Rightarrow$ $A[\bar{B} / \bar{P}]$, where now $\bar{B}$ denote arbitrary formulas in the language of $\mathcal{U}(\mathrm{NFA})$, but $A[\bar{P}]$ is required to be a formula in the language of $\mathcal{U}_{0}$ (NFA). This last restriction is due to the fact that predicates in general depend on the predicate parameters $\bar{P}$. Finally, we obtain an intermediate predicate unfolding system $\mathcal{U}_{1}$ (NFA) by omitting Axiom (16), that is, $\mathcal{U}_{1}$ (NFA) is just $\mathcal{U}(\mathrm{NFA})$ without the Join predicate.

To state the proof-theoretic strength of the three unfolding systems $\mathcal{U}_{0}$ (NFA), $\mathcal{U}_{1}(\mathrm{NFA})$, and $\mathcal{U}(\mathrm{NFA})$, as usual we let $\mathrm{RA}_{<\alpha}$ denote the system of ramified analysis in levels less than $\alpha$. In addition, $\Gamma_{0}$ is the so-called Feferman-Schütte ordinal, which was identified in the early sixties as the limiting number of predicative provability. As in Feferman \& Strahm (2000) we obtain the following proof-theoretic equivalences. In particular, the full unfolding of nonfinitist arithmetic is equivalent to predicative analysis.

11 Observe that nat is alternatively definable from the remaining predicate axioms by $x \in$ nat $\leftrightarrow$ $(\exists y \in \mathrm{N})(x=y)$. 
THEOREM 2.1. We have the following proof-theoretic equivalences:

$$
\begin{aligned}
& \text { 1. } \mathcal{U}_{0}(\mathrm{NFA}) \equiv \mathrm{PA} . \\
& \text { 2. } \mathcal{U}_{1}(\mathrm{NFA}) \equiv \mathrm{RA}_{<\omega} . \\
& \text { 3. } \mathcal{U}(\mathrm{NFA}) \equiv \mathrm{RA}_{<\Gamma_{0}} .
\end{aligned}
$$

In each case we have conservation with respect to arithmetic statements of the system on the left over the system on the right.

The proof of this theorem proceeds in a way that is very similar to the proof-theoretic treatment of the unfolding systems presented in Feferman \& Strahm (2000). However, the argument for the present systems is even simpler since, in contrast to Feferman \& Strahm (2000), we no longer have to deal with least fixed point recursion.

Let us close this section by mentioning a possible generalization of the substitution rule (Subst). Instead of substituting formulas $\bar{B}$ for $\bar{P}$ in derived assertions $A[\bar{P}]$, one could consider the substitution of arbitrary formulas for free predicates in derived rules $A_{1}[\bar{P}], \ldots, A_{n}[\bar{P}] \Rightarrow A[\bar{P}]$, yielding new rules of inference $A_{1}[\bar{B} / \bar{P}], \ldots, A_{n}[\bar{B} / \bar{P}] \Rightarrow$ $A[\bar{B} / \bar{P}]$. Indeed, this generalization (Subst') of (Subst) does not increase the prooftheoretic strength of all three unfolding systems for NFA, as a rather straightforward adaptation of the upper bound arguments in Feferman \& Strahm (2000) and Strahm (2000) reveals. On the other hand, we need to take a form of (Subst') as the basic substitution rule in the formulation of the unfolding systems for finitist arithmetic in the following sections.

§3. The unfolding of FA. We begin here by defining the operational unfolding $\mathcal{U}_{0}(\mathrm{FA})$ of finitist arithmetic FA. That system tells us which operations from and to natural numbers, and which principles concerning them, ought to be accepted if we have accepted FA. It will be shown that Skolem's system PRA of Primitive Recursive Arithmetic is contained in $\mathcal{U}_{0}(\mathrm{FA})$. Later we will see that the operational and even the full unfolding of finitist arithmetic do not go beyond PRA.

3.1. Defining $\mathcal{U}_{0}(\mathrm{FA})$. Large parts of the unfolding systems for FA and NFA are identical. Therefore, we will confine ourselves in the sequel to mentioning the main differences in the definition of the unfolding systems for FA, beginning with its operational unfolding.

The terms of $\mathcal{U}_{0}$ (FA) are the same as the terms of $\mathcal{U}_{0}$ (NFA). Recall that FA is based on the logical operations $\wedge, \vee$, and $\exists$. Accordingly, the formulas of $\mathcal{U}_{0}(\mathrm{FA})$ are generated from the atomic formulas $\perp, s \downarrow,(s=t), \mathrm{N}(s)$, and $P(\bar{s})$ by means of $\wedge, \vee$, and $\exists$; here $P$ denotes an arbitrary free predicate variable of appropriate arity.

The underlying calculus of $\mathcal{U}_{0}(\mathrm{FA})$ is a Gentzen-type sequent system based on sequents of the form $\Gamma \rightarrow A$ for $\Gamma$ being a finite sequence of formulas in the language of $\mathcal{U}_{0}(\mathrm{FA})$. In case $\Gamma$ is empty, we will write $A$ for $\rightarrow A$. The logical axioms and rules of inference are the standard ones: apart from identity axioms, rules for $\perp$, cut and structural rules, these include the usual Gentzen-type rules for $\wedge$ and $\vee$ as well as introduction of $\exists$ on the left and on the right in the form

$$
\frac{\Gamma \rightarrow A[t] \wedge t \downarrow}{\Gamma \rightarrow(\exists x) A[x]}, \quad \frac{\Gamma, A[u] \rightarrow B}{\Gamma,(\exists x) A[x] \rightarrow B} \quad(u \text { fresh })
$$

Note that quantifiers range over defined objects only. Moreover, defined terms can be substituted for free variables according to the following rule of inference; here $\Gamma[t]$ stands for the sequence $(B[t]: B[u] \in \Gamma)$. 


$$
\frac{\Gamma[u] \rightarrow A[u]}{\Gamma[t], t \downarrow \rightarrow A[t]}
$$

Finally, the equality and strictness axioms of our underlying logic of partial terms are given a Gentzen-style formulation in the obvious way.

The nonlogical axioms and rules of $\mathcal{U}_{0}(\mathrm{FA})$ include the relativization of the axioms and rules of FA to the predicate $\mathrm{N}$, namely

(1) $u \in \mathrm{N}, u^{\prime}=0 \rightarrow \perp$

(2) $u \in \mathrm{N} \rightarrow \operatorname{Pd}\left(u^{\prime}\right)=u$

(3) $\frac{\Gamma \rightarrow P(0) \quad \Gamma, u \in \mathrm{N}, P(u) \rightarrow P\left(u^{\prime}\right)}{\Gamma, v \in \mathrm{N} \rightarrow P(v)}$,

as well as suitable formulations of the Axioms (2)-(8) of $\mathcal{U}_{0}$ (NFA). We will not spell out these axioms again, but instead give an example how to reformulate Axiom (4) about the $S$ combinator in our new setting. This now breaks into the following two axioms,

$$
\mathrm{s} a b \downarrow \quad \text { and } \quad \mathrm{s} a b c \downarrow \vee a c(b c) \downarrow \rightarrow \mathrm{s} a b c=a c(b c) .
$$

What is still missing in $\mathcal{U}_{0}(\mathrm{FA})$ is a suitable version of the substitution rule (Subst), which is central to all unfolding systems. In order to fit this into our Gentzen-style setting, (Subst) has to be formulated in a somewhat more general form. For that purpose, we let $\Sigma, \Sigma_{1}, \Sigma_{2}, \ldots$ range over sequents in the language of $\mathcal{U}_{0}(\mathrm{FA})$. A rule of inference for such sequents has the general form

$$
\frac{\Sigma_{1}, \Sigma_{2}, \ldots, \Sigma_{n}}{\Sigma}
$$

which we simply abbreviate by $\Sigma_{1}, \Sigma_{2}, \ldots, \Sigma_{n} \Rightarrow \Sigma$ in the sequel; we also allow $n$ to be 0 , that is, rules with an empty list of premises are possible. As usual we call a rule of inference $\Sigma_{1}, \Sigma_{2}, \ldots, \Sigma_{n} \Rightarrow \Sigma$ derivable from a collection of axioms and rules $\mathcal{T}$ (all in Gentzen-style), if the sequent $\Sigma$ is derivable from $\mathcal{T} \cup\left\{\Sigma_{1}, \Sigma_{2}, \ldots, \Sigma_{n}\right\}$.

In the following $\bar{P}=P_{1}, \ldots, P_{m}$ denotes a finite sequence of free predicate symbols of finite arity and $\bar{B}=B_{1}, \ldots, B_{m}$ a corresponding sequence of formulas in the language of $\mathcal{U}_{0}(\mathrm{FA})$. If $\Sigma[\bar{P}]$ is a sequent possibly containing the free predicates $\bar{P}$, then above $\Sigma[\bar{B} / \bar{P}]$ denotes the sequent $\Sigma[\bar{P}]$ with each subformula of the form $P_{i}(\bar{t})$ replaced by $(\exists \bar{x})(\bar{t}=\bar{x} \wedge B[\bar{x}])$, where the length of $\bar{x}$ is equal to the arity of $P_{i}$.

We are now ready to state our (meta) substitution rule (Subst'). Its meaning is as follows: whenever the axioms and rules of inference at hand allow us to show that the rule $\Sigma_{1}, \Sigma_{2}, \ldots, \Sigma_{n} \Rightarrow \Sigma$ is derivable, then we can adjoin each of its substitution instances $\Sigma_{1}[\bar{B} / \bar{P}], \Sigma_{2}[\bar{B} / \bar{P}], \ldots, \Sigma_{n}[\bar{B} / \bar{P}] \Rightarrow \Sigma[\bar{B} / \bar{P}]$ as a new rule of inference to $\mathcal{U}_{0}$ (FA), for $B_{i}[\bar{x}]$ being formulas in the language of $\mathcal{U}_{0}(\mathrm{FA}) .{ }^{12}$ Symbolically,

$$
\frac{\Sigma_{1}, \Sigma_{2}, \ldots, \Sigma_{n} \Rightarrow \Sigma}{\Sigma_{1}[\bar{B} / \bar{P}], \Sigma_{2}[\bar{B} / \bar{P}], \ldots, \Sigma_{n}[\bar{B} / \bar{P}] \Rightarrow \Sigma[\bar{B} / \bar{P}]} .
$$

Observe that derivability of rules is a dynamic process as we unfold FA. In particular, new rules of inference obtained by (Subst') allow us to establish new derivable rules, to which

12 In the sequel, when substituting formulas in rules of inference, we tacitly assume that free variables in $\Sigma_{1}, \Sigma_{2}, \ldots, \Sigma_{n}$ which are eigenvariables in the derivation of $\Sigma$ from $\Sigma_{1}, \Sigma_{2}, \ldots, \Sigma_{n}$ do not occur in $\bar{B}$. Such variables, by definition, are exactly the eigenvariables of the new rule of inference $\Sigma_{1}[\bar{B} / \bar{P}], \Sigma_{2}[\bar{B} / \bar{P}], \ldots, \Sigma_{n}[\bar{B} / \bar{P}] \Rightarrow \Sigma[\bar{B} / \bar{P}]$. 
in turn we can apply (Subst'). In particular, the usual rule of induction

$$
\frac{\Gamma \rightarrow A[0] \quad \Gamma, u \in \mathrm{N}, A[u] \rightarrow A\left[u^{\prime}\right]}{\Gamma, v \in \mathrm{N} \rightarrow A[v]}
$$

is an immediate consequence of (Subst') applied to Rule (3) of FA. Moreover, the substitution rule in its usual form as stated in Section 2,

$$
\frac{\Sigma[\bar{P}]}{\Sigma[\bar{B} / \bar{P}]}
$$

is readily seen to be an admissible rule of inference of $\mathcal{U}_{0}(\mathrm{FA})$. Finally, note that we could have also stated the logical rules of $\mathcal{U}_{0}(\mathrm{FA})$ mentioned above using free predicate variables and then derive substitution instances thereof using the rule (Subst').

\section{2. $\mathcal{U}_{0}(\mathrm{FA})$ contains $\mathrm{PRA}$.}

LEMMA 3.1. All primitive recursive functions can be introduced in $\mathcal{U}_{0}(\mathrm{FA})$.

Proof. We inductively assign terms to each (description of a) primitive recursive function and show that these terms provably define a total number-theoretic function in $\mathcal{U}_{0}$ (FA). The crucial step is to show closure under primitive recursion. Hence, let $r$ and $s$ be terms taking number arguments $(\bar{u})$ and $(\bar{u}, v, w)$, respectively, and assume that $r$ and $s$ have been shown to be total in $\mathcal{U}_{0}(\mathrm{FA})$. Then we can make use of the recursion or fixed point theorem and definition by cases on $\mathrm{N}$ to define a term $t$ so that provably in $\mathcal{U}_{0}(\mathrm{FA})$,

$$
t(\bar{u}, 0) \simeq r(\bar{u}) \text { and } t\left(\bar{u}, v^{\prime}\right) \simeq s(\bar{u}, v, t(\bar{u}, v)),
$$

for all $\bar{u}, v$ in $\mathrm{N}$. Here the partial equality relation $\simeq$ is understood as before. We have noted above that the usual rule of induction is available in $\mathcal{U}_{0}(\mathrm{FA})$ as a consequence of (Subst'). Hence, to show that $t(\bar{u}, v) \in \mathrm{N}$ in $\mathcal{U}_{0}$ (FA) we just need to establish

$$
\bar{u} \in \mathrm{N} \rightarrow t(\bar{u}, 0) \in \mathrm{N} \text { and } \bar{u} \in \mathrm{N}, v \in \mathrm{N}, t(\bar{u}, v) \in \mathrm{N} \rightarrow t\left(\bar{u}, v^{\prime}\right) \in \mathrm{N} .
$$

But this is immediate from the recursion equations above and the fact that $r$ and $s$ are already known to define total functions on $\mathrm{N}$.

The following is an immediate consequence.

TheOrem 3.2. Primitive Recursive Arithmetic PRA is contained in $\mathcal{U}_{0}(\mathrm{FA})$.

In the next subsection, it will be shown that this lower bound for $\mathcal{U}_{0}(\mathrm{FA})$ is indeed the best possible one.

3.3. $\mathcal{U}_{0}(\mathrm{FA})$ does not go beyond PRA. To obtain the upper bound result we turn to a recursion-theoretic interpretation of $\mathcal{U}_{0}(\mathrm{FA})$ into a version $\Sigma_{1}^{+}$-IA of PRA plus $\Sigma_{1}$ induction. In $\Sigma_{1}^{+}$-IA we allow positive occurrences of the free relation symbols $\bar{P}$ in $\Sigma_{1}$ formulas for which complete induction is permitted. Special attention has to be given in this setting to the translation of the substitution rule (Subst') of $\mathcal{U}_{0}(\mathrm{FA})$.

In the sequel we let $\mathcal{L}_{1}$ denote the usual language of arithmetic with number variables $a, b, c, u, w, v, x, y, z, \ldots$ as well as function and relation symbols for all primitive recursive functions and relations. Moreover, it is assumed that $\mathcal{L}_{1}$ contains free relation symbols $\bar{P}$. Terms $(r, s, t, \ldots)$ and formulas $(A, B, C, \ldots)$ of $\mathcal{L}_{1}$ are defined as usual. A formula of $\mathcal{L}_{1}$ is called $\Delta_{0}^{+}$if (i) all its quantifiers are bounded and (ii) the relation symbols $\bar{P}$ only have positive occurrences in it. Accordingly, the $\Sigma_{1}^{+}$formulas of $\mathcal{L}_{1}$ are obtained 
by prefixing existential quantifiers to $\Delta_{0}^{+}$formulas. $\Pi_{1}^{+}$and $\Pi_{2}^{+}$formulas are defined analogously.

The system $\Sigma_{1}^{+}$-IA is defined to be the first order $\mathcal{L}_{1}$ theory which includes the defining axioms for the function and relation symbols of $\mathcal{L}_{1}$ as well as the schema of complete induction,

$$
A[0] \wedge(\forall x)\left(A[x] \rightarrow A\left[x^{\prime}\right]\right) \rightarrow(\forall x) A[x]
$$

for all $\Sigma_{1}^{+}$formulas of the language $\mathcal{L}_{1}$. Observe that $\Sigma_{1}^{+}$-IA does not otherwise include axioms for the free relations $\bar{P}$ (apart from equality axioms, of course). Hence, if $\Sigma_{1}$-IA denotes the usual system of arithmetic with $\Sigma_{1}$ induction in the language $\mathcal{L}_{1}$ without $\bar{P}$, then we immediately have that $\Sigma_{1}^{+}$-IA is a conservative extension of $\Sigma_{1}$-IA. Moreover, it is well known (cf., e.g., Mints, 1973, or Parsons, 1970) that $\Sigma_{1}-$ IA is a conservative extension of Primitive Recursive Arithmetic PRA in the following sense: if $\Sigma_{1}$-IA proves $(\forall \bar{x})(\exists y) A[\bar{x}, y]$ for $A$ a quantifier-free formula in the language $\mathcal{L}_{1}$, then there exists a (symbol of a) primitive recursive function $f$ such that PRA proves $A[\bar{x}, f(\bar{x})]$.

Let us now turn to the interpretation of $\mathcal{U}_{0}(\mathrm{FA})$ in $\Sigma_{1}^{+}$-IA. The applicative part of $\mathcal{U}_{0}(\mathrm{FA})$ is simply modeled in terms of ordinary recursion theory so that $(a \cdot b)$ translates into $\{a\}(b)$ in $\mathcal{L}_{1}$, where $\{n\}$ for $n=0,1,2, \ldots$ is a standard enumeration of the partial recursive functions. Then it is possible to assign pairwise different numerals to the constants sc, pd, k, s, p, $\mathrm{p}_{0}, \mathrm{p}_{1}, \mathrm{~d}, \mathrm{t}$, ff, and e so that the corresponding applicative axioms are satisfied.

More formally, for each term $t$ in the language of $\mathcal{U}_{0}(\mathrm{FA})$ there exists a formula $\operatorname{Val}_{t}[a]$ expressing that $a$ is the value of $t$ under the interpretation described above. It is easily seen that $\operatorname{Val}_{t}[a]$ is (equivalent to) a $\Sigma_{1}$ formula of $\mathcal{L}_{1}$. Accordingly, the atomic formulas $\perp, t \downarrow$, $(s=t), \mathrm{N}(t)$, and $P(\bar{t})$ are given their obvious interpretations in $\mathcal{L}_{1}$ with the translation of $\mathrm{N}$ ranging over all natural numbers. Thus, the translation of these atomic formulas is (equivalent to) a $\Sigma_{1}^{+}$formula of $\mathcal{L}_{1}$.

We now have for each formula $A$ in the language of $\mathcal{U}_{0}(\mathrm{FA})$ its natural translation $A^{*}$ in $\mathcal{L}_{1}$, containing the same parameters as $A$. It is readily seen that $A^{*}$ is provably equivalent in $\Sigma_{1}^{+}$-IA to a $\Sigma_{1}^{+}$formula; in particular, the free relations $\bar{P}$ only occur positively in $A^{*}$. The translation $*$ is extended to sequents $\Gamma \rightarrow B$ in the obvious way, by taking $(\Gamma \rightarrow B)^{*}$ to be $(\bigwedge \Gamma)^{*} \rightarrow B^{*}$. Finally, the $*$ translation of a rule of inference is understood accordingly.

\section{LEMMA 3.3.}

1. The * translation of the induction rule (3) of $\mathrm{FA}$ is a derivable rule of $\Sigma_{1}^{+}-\mathrm{IA}$.

2. The $*$ translation of each axiom of $\mathcal{U}_{0}(\mathrm{FA})$ is derivable in $\Sigma_{1}^{+}-\mathrm{IA}$.

Proof. The first assertion is obvious since the formulas $P(u)$ are $\Sigma_{1}^{+}$formulas of $\mathcal{L}_{1}$ and, hence, the induction axiom of $\Sigma_{1}^{+}$-IA can be used to show the derivability of the * translation of the induction rule.

For 2, the translation of the applicative axioms of $\mathcal{U}_{0}(\mathrm{FA})$ is immediate.

The final step in our verification that $\mathcal{U}_{0}(\mathrm{FA})$ is contained in $\Sigma_{1}^{+}$-IA via our translation $*$ consists in the treatment of the substitution rule $\left(\mathrm{Subst}^{\prime}\right)$ of $\mathcal{U}_{0}(\mathrm{FA})$. For that purpose we have to consider derivable rules of $\Sigma_{1}^{+}-\mathrm{IA}$, which have the general form

$$
\frac{A_{1}, A_{2}, \ldots, A_{n}}{A}
$$


for $A_{1}, A_{2}, \ldots, A_{n}, A$ being formulas in the language $\mathcal{L}_{1}$; as above we will use the more compact notation $A_{1}, A_{2}, \ldots A_{n} \Rightarrow A$. A first simple observation tacitly used in the following is that if we are given rules $\mathcal{R}_{1}, \mathcal{R}_{2}, \ldots, \mathcal{R}_{n}$ which are derivable in $\Sigma_{1}^{+}$-IA and, in addition, we know that the rule $\mathcal{R}$ is derivable in $\Sigma_{1}^{+}$-IA augmented by the rules $\mathcal{R}_{1}, \mathcal{R}_{2}, \ldots, \mathcal{R}_{n}$, then of course $\mathcal{R}$ is already a derivable rule of $\Sigma_{1}^{+}$-IA alone.

The crucial fact in the treatment of (Subst') is the following: if $A[\bar{P}]$ is a $\Sigma_{1}^{+}$formula of $\mathcal{L}_{1}$ and if $\bar{B}$ consists of $\Sigma_{1}^{+}$formulas, then $A[\bar{B} / \bar{P}]$ is provably equivalent in $\Sigma_{1}^{+}$-IA to a $\Sigma_{1}^{+}$formula. As an immediate consequence we obtain that derivable rules of $\Sigma_{1}^{+}$-IA are closed under substitutions with respect to $\Sigma_{1}^{+}$formulas.

LEMMA 3.4.

1. If $A$ and $\bar{B}$ are $\Sigma_{1}^{+}$formulas of $\mathcal{L}_{1}$, then $A[\bar{B} / \bar{P}]$ is provably equivalent in $\Sigma_{1}^{+}-\mathrm{IA}$ to a $\Sigma_{1}^{+}$formula.

2. If $A_{1}, A_{2}, \ldots, A_{n} \Rightarrow A$ is a derivable rule of $\Sigma_{1}^{+}-\mathrm{IA}$ and $\bar{B}$ are $\Sigma_{1}^{+}$formulas of $\mathcal{L}_{1}$, then also $A_{1}[\bar{B} / \bar{P}], A_{2}[\bar{B} / \bar{P}], \ldots, A_{n}[\bar{B} / \bar{P}] \Rightarrow A[\bar{B} / \bar{P}]$ is a derivable rule of $\Sigma_{1}^{+}$-IA.

Proof. The first assertion of our lemma is a simple consequence of $\Sigma_{1}^{+}$collection, which is available in $\Sigma_{1}^{+}$-IA (cf., e.g., Sieg, 1985, p. 53).

For the second assertion let us assume that $A_{1}, A_{2}, \ldots, A_{n} \Rightarrow A$ is derivable in $\Sigma_{1}^{+}$-IA and $\bar{B}$ is in $\Sigma_{1}^{+}$. Then we have a proof of $A$ in $\Sigma_{1}^{+}$-IA plus $A_{1}, A_{2}, \ldots, A_{n}$. Replacing $\bar{P}$ by $\bar{B}$ in this proof yields a proof of $A[\bar{B} / \bar{P}]$ in $\Sigma_{1}^{+}$-IA augmented by the axioms $A_{1}[\bar{B} / \bar{P}], A_{2}[\bar{B} / \bar{P}], \ldots, A_{n}[\bar{B} / \bar{P}]$; here one uses the first assertion of this lemma to show that possible instances of $\Sigma_{1}^{+}$induction in $\Sigma_{1}^{+}$-IA again carry over to instances of $\Sigma_{1}^{+}$ induction if $\bar{B}$ is substituted for $\bar{P}$.

From the previous two lemmas we now immediately obtain that the $*$ translations of the axioms and rules of inference of $\mathcal{U}_{0}(\mathrm{FA})$ are derivable in $\Sigma_{1}^{+}-\mathrm{IA}$. As a consequence, the $*$ translation of a derivable formula of $\mathcal{U}_{0}(\mathrm{FA})$ is provable in $\Sigma_{1}^{+}-\mathrm{IA}$. To summarize:

THEOREM 3.5. $\mathcal{U}_{0}(\mathrm{FA})$ is contained in $\Sigma_{1}^{+}$-IA via the translation $*$.

Using the result about the $\Pi_{2}$ conservativity of $\Sigma_{1}^{+}$-IA over PRA mentioned above and Theorem 3.2 we thus have the following:

COROLlary 3.6. $\mathcal{U}_{0}(\mathrm{FA})$ is proof-theoretically equivalent to Primitive Recursive Arithmetic PRA.

In particular, we have that provably total terms of type $\left(\mathrm{N}^{n} \rightarrow \mathrm{N}\right)$ give rise to primitive recursive algorithms.

COROLlary 3.7. Let $t$ be a closed $\mathcal{U}_{0}(\mathrm{FA})$ term and assume that $\mathcal{U}_{0}(\mathrm{FA})$ proves the sequent $\bar{u} \in \mathrm{N} \rightarrow t(\bar{u}) \in \mathrm{N}$. Then $t$ defines a primitive recursive function.

3.4. Defining $\mathcal{U}(\mathrm{FA})$. The full unfolding $\mathcal{U}(\mathrm{FA})$ of finitist arithmetic $\mathrm{FA}$ is an extension of the operational unfolding $\mathcal{U}_{0}(\mathrm{FA})$ and is used, in addition, to answer the question of which operations on and to predicates, and which principles concerning them, are to be accepted if one has accepted FA. It is proved in this section that $\mathcal{U}(\mathrm{FA})$ does not go beyond Primitive Recursive Arithmetic PRA in proof-theoretic strength.

The language of $\mathcal{U}(\mathrm{FA})$ is an extension of the language of $\mathcal{U}_{0}(\mathrm{FA})$. It includes, in addition, the constants nat (natural numbers), eq (equality), $\operatorname{pr}_{P}$ (free predicate $P$ ), inv 
(inverse image), conj (conjunction), disj (disjunction), ex (existential quantification), and join (disjoint unions). Moreover, as above, we have a new unary relation symbol $\Pi$ for (codes of) predicates and a binary relation symbol $\in$ for the elementhood relation. The terms of $\mathcal{U}(\mathrm{FA})$ are built as before. The formulas of $\mathcal{U}(\mathrm{FA})$ extend the formulas of $\mathcal{U}_{0}(\mathrm{FA})$ by allowing the new atomic formulas $\Pi(t)$ and $s \in t$.

The axioms of $\mathcal{U}(\mathrm{FA})$ extend those of $\mathcal{U}_{0}(\mathrm{FA})$. In addition, we have the obvious defining axioms for the basic predicates of $\mathcal{U}(\mathrm{FA})$. These include straightforward reformulations using sequents of the Axioms (9)-(12) and (14) of $\mathcal{U}(\mathrm{NFA})$ as well as the expected axiom about existentially quantified predicates; just to give an example, the latter axiom is spelled out in our restricted logical setting by the following three sequents.

$$
\begin{gathered}
\Pi(a) \rightarrow \Pi(\operatorname{ex}(a)), \\
\Pi(a), u \in \operatorname{ex}(a) \rightarrow(\exists x \in \mathrm{N})((u, x) \in a), \text { and } \\
\Pi(a),(\exists x \in \mathrm{N})((u, x) \in a) \rightarrow u \in \operatorname{ex}(a) .
\end{gathered}
$$

Further, Axiom (16) of $\mathcal{U}$ (NFA) concerning join is now stated in terms of suitable inference rules; this is due to the absence of universal quantification in the framework of finitist arithmetic. Thus, we have the following three rules of inference with $J$ being the formula from Section 2 and $u$ denoting a fresh variable.

$$
\frac{\Gamma, u \in \mathrm{N} \rightarrow \Pi(t u)}{\Gamma \rightarrow \Pi(\operatorname{join}(t))}, \quad \frac{\Gamma, u \in \mathrm{N} \rightarrow \Pi(t u)}{\Gamma, v \in \operatorname{join}(t) \rightarrow J[t, v]}, \quad \frac{\Gamma, u \in \mathrm{N} \rightarrow \Pi(t u)}{\Gamma, J[t, v] \rightarrow v \in \operatorname{join}(t)} .
$$

Finally, $\mathcal{U}(\mathrm{FA})$ of course also includes the substitution rule (Subst') which we have spelled out for $\mathcal{U}_{0}(\mathrm{FA})$. The formulas $\bar{B}$ to be substituted for $\bar{P}$ are now in the language of $\mathcal{U}(\mathrm{FA})$; the rule in the premise of $\left(\right.$ Subst $\left.^{\prime}\right)$, however, is required to be in the language of $\mathcal{U}_{0}$ (FA). This last restriction is imposed as before since predicates may depend on the free relation symbols $\bar{P}$. The intermediate unfolding system $\mathcal{U}_{1}(\mathrm{FA})$ for FA is obtained by dropping the rules about join.

3.5. $\mathcal{U}(\mathrm{FA})$ is contained in $\Sigma_{1}^{+}-\mathrm{IA}$. In this section we will extend the embedding of $\mathcal{U}_{0}(\mathrm{FA})$ into $\Sigma_{1}^{+}$-IA by providing a suitable interpretation of the predicate part of $\mathcal{U}(\mathrm{FA})$.

We here make use of the usual primitive recursive coding machinery: $\langle\ldots\rangle$ is a standard primitive recursive function for forming $n$-tuples $\left\langle t_{1}, \ldots, t_{n}\right\rangle$; Seq denotes the primitive recursive set of sequence numbers; $\ln (t)$ denotes the length of (the sequence coded by) $t$; $\operatorname{Seq}_{n}(t)$ abbreviates $\operatorname{Seq}(t) \wedge \operatorname{lh}(t)=n ;(t)_{i}$ is the $i$ th component of (the sequence coded by) $t$ for $i<\mathrm{lh}(t)$, in particular, $t=\left\langle(t)_{0}, \ldots,(t)_{\operatorname{lh}(t)-1}\right\rangle$ for sequence numbers $t$; we write $\operatorname{lst}(t)$ for $\ln (t)-1$ and $(t)_{i, j}$ instead of $\left((t)_{i}\right)_{j} ; \star$ denotes the usual primitive recursive operation of sequence concatenation; finally, if $\bar{x}=x_{1}, \ldots, x_{n}$ then we write $\langle\bar{x}\rangle$ for $\left\langle x_{1}, \ldots, x_{n}\right\rangle$.

For the interpretation of the predicates of $\mathcal{U}(\mathrm{FA})$ and their extension we could basically just consider the recursively enumerable sets, which have all the required closure properties. Intuitively, the reason that join does not increase strength is that the recursive join of $\Sigma_{1}$ predicates is $\Sigma_{1}$. This is in contrast to the case of NFA, where the full unfolding also recognizes as defined predicates expressed by certain infinitary formulas which cannot be reduced to ordinary arithmetical formulas.

But since predicates in $\mathcal{U}(\mathrm{FA})$ depend on the free relations $\bar{P}$, the situation is slightly delicate, especially because we will have to take care of positive and negative occurrences of $\bar{P}$ and possible substitution instances in the sequel. For that reason we follow a more direct approach and define the extension of (codes of) predicates by using a suitable 
notion of computation sequence. In the sequel we will only model a finite collection $\bar{P}=P_{1}, \ldots, P_{n}$ of free relation symbols for some fixed natural number $n$. This is no essential restriction since a fixed derivation in $\mathcal{U}(\mathrm{FA})$ may only use finitely many free relation symbols.

Below we use the following codes for predicates $\left(\bar{P}=P_{1}, \ldots, P_{n}\right)$ :

- $\langle 0\rangle$ for the predicate nat,

- $\langle 1\rangle$ for the predicate eq,

- $\langle 2, i\rangle$ for the predicate $\operatorname{pr}_{P_{i}}$,

- $\langle 3, b, c\rangle$ for the predicate inv $(b, c)$,

- $\langle 4, b, c\rangle$ for the predicate $\operatorname{conj}(b, c)$,

- $\langle 5, b, c\rangle$ for the predicate $\operatorname{disj}(b, c)$,

- $\langle 6, b\rangle$ for the predicate ex $(b)$,

- $\langle 7, b\rangle$ for the predicate join $(b)$.

The extension of these predicate codes will be determined by using the notion $\Pi$ Seq $[a]$ of a predicate computation sequence. Informally, ПSeq[a] means that $a$ is a sequence number whose entries are pairs $\langle x, y\rangle$ witnessing that $y$ belongs to the extension of the predicate code $x$; the sequence $a$ can be thought of as a proof of the fact that $y$ belongs to $x$ for each such pair $\langle x, y\rangle$. Accordingly, we set

$$
\Pi \operatorname{Seq}[a]:=\operatorname{Seq}(a) \wedge(\forall u<\operatorname{lh}(a))\left[\operatorname{Seq}_{2}\left((a)_{u}\right) \wedge C\left[(a)_{u, 0},(a)_{u, 1}, a, u\right]\right],
$$

where $C[x, y, a, u]$ is the disjunction of the following formulas (0)-(7): ${ }^{13}$

(0) $x=\langle 0\rangle$,

(1) $x=\langle 1\rangle \wedge(\exists z)(y=\langle z, z\rangle)$,

(2) $\bigvee_{i=1}^{n}\left(x=\langle 2, i\rangle \wedge P_{i}(\tilde{y})\right)$,

(3) $(\exists b, c)\left[x=\langle 3, b, c\rangle \wedge(\exists v<u)\left((a)_{v}=\langle b,\{c\}(y)\rangle\right)\right]$,

(4) $(\exists b, c)\left[x=\langle 4, b, c\rangle \wedge(\exists v, w<u)\left((a)_{v}=\langle b, y\rangle \wedge(a)_{w}=\langle c, y\rangle\right)\right]$,

(5) $(\exists b, c)\left[x=\langle 5, b, c\rangle \wedge(\exists v<u)\left((a)_{v}=\langle b, y\rangle \vee(a)_{v}=\langle c, y\rangle\right)\right]$,

(6) $(\exists b)\left[x=\langle 6, b\rangle \wedge(\exists v<u)(\exists z)\left((a)_{v}=\langle b,\langle y, z\rangle\rangle\right)\right]$,

(7) $(\exists b)\left[x=\langle 7, b\rangle \wedge(\exists v<u)\left(\exists y_{0}, y_{1}\right)\left(y=\left\langle y_{0}, y_{1}\right\rangle \wedge(a)_{v}=\left\langle\{b\}\left(y_{0}\right), y_{1}\right\rangle\right)\right]$.

Observe that the free predicate symbols $\bar{P}$ only have positive occurrences in the $\mathcal{L}_{1}$ formula $\Pi$ Seq $[a]$. Moreover, since $\Sigma_{1}^{+}$collection is available in $\Sigma_{1}^{+}$-IA, we have that $\Pi S e q[a]$ is equivalent to a $\Sigma_{1}^{+}$formula of $\mathcal{L}_{1}$, provably in $\Sigma_{1}^{+}$-IA.

Now to translate the predicate part of $\mathcal{U}(\mathrm{FA})$, let $\Pi$ range over the set of natural numbers and translate the formula $(y \in x)$ in the language of $\mathcal{U}(\mathrm{FA})$ by the $\mathcal{L}_{1}$ formula $\mathrm{E}[y, x]$,

$$
\mathrm{E}[y, x]:=(\exists a)\left[\Pi \operatorname{Seq}[a] \wedge(a)_{\operatorname{lst}(a)}=\langle x, y\rangle\right] .
$$

More generally, formulas of the form $(s \in t)$ are translated accordingly, using the formulas $\mathrm{Val}_{s}[a]$ and $\mathrm{Val}_{t}[a]$. Observe that if $x$ is not the code of a predicate of the forms specified above, then the extension of $x$ is empty according to the definition of $\mathrm{E}$. Moreover, we have that $\mathrm{E}[y, x]$ is also a $\Sigma_{1}^{+}$formula of the language $\mathcal{L}_{1}$.

13 For a free $n$-ary predicate symbol $P_{i}$, we let $P_{i}(\tilde{y})$ stand for $P_{i}\left((y)_{0}, \ldots,(y)_{n-1}\right)$ if $n \geq 2, P_{i}(y)$ if $n=1$ and $P_{i}$ in case $n=0$. 
We now have for each formula $A$ in the language of $\mathcal{U}(\mathrm{FA})$ its natural translation $A^{*}$ in $\mathcal{L}_{1}$, containing the same parameters as $A$. Clearly, $A^{*}$ is provably equivalent in $\Sigma_{1}^{+}$-IA to a $\Sigma_{1}^{+}$formula; in particular, the free relations $\bar{P}$ only occur positively in $A^{*}$. The translation $*$ is extended to sequents in the obvious way, and the $*$ translation of a rule of inference is understood accordingly.

LEMMA 3.8. The $*$ translation of each axiom of $\mathcal{U}(\mathrm{FA})$ is derivable in $\Sigma_{1}^{+}-\mathrm{IA}$.

Proof. The axioms about predicates are direct by construction. To give an example, consider the axioms about existential quantification. To this end, let us reason informally in $\Sigma_{1}^{+}-\mathrm{IA}$ and first assume that $\mathrm{E}[y,\langle 6, x\rangle]$. Hence, there exists a predicate computation sequence $a$ such that $(a)_{\mid \operatorname{st}(a)}=\langle\langle 6, x\rangle, y\rangle$. By the definition of $\Pi$ Seq $[a]$ this yields some $v<\operatorname{Ist}(a)$ and a $z$ with $(a)_{v}=\langle x,\langle y, z\rangle\rangle$. This shows that the sequence $\left\langle(a)_{0}, \ldots,(a)_{v}\right\rangle$ witnesses $\mathrm{E}[\langle y, z\rangle, x]$. For the reverse direction assume that there is a number $z$ such that $\mathrm{E}[\langle y, z\rangle, x]$. Then there is a predicate computation sequence $a$ with $(a)_{\mid \operatorname{st}(a)}=\langle x,\langle y, z\rangle\rangle$. Now clearly $a \star\langle\langle\langle 6, x\rangle, y\rangle\rangle$ witnesses $\mathrm{E}[y,\langle 6, x\rangle]$.

Next, note that our model of $\mathcal{U}(\mathrm{FA})$ in $\Sigma_{1}^{+}-\mathrm{IA}$ in fact validates a stronger form of the Join axiom as is claimed by the corresponding rules of inference of $\mathcal{U}(\mathrm{FA})$. Indeed, our modeling takes into account predicates of the form join $(f)$ which do not satisfy the premise $(\forall x \in \mathrm{N}) \Pi(f x)$. Of course, the reason that one can easily accommodate this strengthening lies in the absence of negated predicates.

Finally, the treatment of the substitution rule $\left(\right.$ Subst $\left.^{\prime}\right)$ is the same as for $\mathcal{U}_{0}(\mathrm{FA})$ so that we can now state the following:

THEOREM 3.9. $\mathcal{U}(\mathrm{FA})$ is contained in $\Sigma_{1}^{+}-\mathrm{IA}$ via the translation $*$.

The following is now an immediate consequence of the result about the $\Pi_{2}$ conservativity of $\Sigma_{1}^{+}$-IA over PRA mentioned above and Theorem 3.2.

COROLlary 3.10. $\mathcal{U}_{0}(\mathrm{FA}), \mathcal{U}_{1}(\mathrm{FA})$, and $\mathcal{U}(\mathrm{FA})$ are all proof-theoretically equivalent to Primitive Recursive Arithmetic PRA.

COROLLARY 3.11. Let $t$ be a closed $\mathcal{U}_{0}(\mathrm{FA})$ term and assume that $\mathcal{U}(\mathrm{FA})$ proves the sequent $\bar{u} \in \mathrm{N} \rightarrow t(\bar{u}) \in \mathrm{N}$. Then $t$ defines a primitive recursive function.

\$4. The unfolding of FA with Bar Rule. The aim of this section is to study a natural Bar Rule BR which allows us to use a special schema of transfinite induction along provably well-founded relations. That allows one to derive a general form of nested recursion along well-founded relations. Then by the well-known result of Tait (1961), it is shown that $\mathcal{U}_{0}(\mathrm{FA})$ augmented by BR proves the well-foundedness of each $\alpha$ less than $\varepsilon_{0}$. Further, an embedding of $\mathcal{U}_{0}(\mathrm{FA})$ plus $\mathrm{BR}$ in $\mathrm{ACA}_{0}$ reveals that this lower bound is sharp. Later we will see that even the full unfolding of FA + BR does not go beyond PA.

By way of background and motivation for our formulation of $B R$, we begin with the Bar Induction Principle (BI) that was introduced by L.E.J. Brouwer in his intuitionistic redevelopment of analysis; see Troelstra \& van Dalen (1988), Ch. 8.4 for an exposition. Roughly speaking BI says that if there are no infinite descending sequences in a given decidable tree, that is, if every potential such sequence meets a "bar", then transfinite induction follows on the tree ordering. This has been taken in both intuitionistic and classical contexts in the more general form that if $\prec$ is a partial ordering satisfying NDS $(\prec)$ (no infinite descending sequence property for $\prec$ ) then the principle $\operatorname{TI}(\prec, P)$ of transfinite 
induction on $\prec$ holds for arbitrary predicates $P$. The corresponding Bar Rule BR is of the form that we may infer $\operatorname{TI}(\prec, P)$ from $\operatorname{NDS}(\prec)$ for each predicate $P$. For our purposes, it is sufficient to restrict this to provably decidable linear orderings $\prec$ in the natural numbers, with 0 as least element. But further restrictions have to be made in order to fit a version of BR to the language of FA. First of all, the statement that a given function $f$ on $\mathrm{N}$ is descending in the $\prec$ relation, as long as it is not 0 , is universal, so cannot be expressed as a formula of our language. Instead, we add a new function constant symbol $f$ interpreted as an arbitrary (or "anonymous") function, and require that we establish a rule, $\operatorname{NDS}(\prec, f)$, that allows us to infer from the hypotheses that $\mathrm{f}: \mathrm{N} \rightarrow \mathrm{N}$ and that $\mathrm{f}\left(u^{\prime}\right) \prec \mathrm{f}(u)$ as long as $\mathrm{f}(u) \neq 0$ (' $u$ ' a free variable) the conclusion $(\exists x \in \mathrm{N})(\mathrm{f}(x)=0)$. In addition, we must modify $\operatorname{TI}(\prec, P)$, since its standard formulation for a unary predicate $P$ is of the form:

$$
(\forall x)[(\forall u \prec x) P(u) \rightarrow P(x)] \rightarrow(\forall x) P(x) .
$$

Again, the idea is to treat this as a rule of the form:

$$
\text { from } \quad(\forall u)[u \prec x \rightarrow P(u)] \rightarrow P(x) \quad \text { infer } \quad P(x) .
$$

But we still need an additional step to reformulate the hypothesis of this rule in the language of FA. For atomic $A, B$ write $A \supset B$ for $(\neg A \vee B)$. Then the hypothesis is implied by

$$
\left[t_{1} \prec x \supset P\left(t_{1}\right)\right] \wedge \cdots \wedge\left[t_{m} \prec x \supset P\left(t_{m}\right)\right] \rightarrow P(x),
$$

where the $t_{i}$ are terms that have been proved to be defined. Now it may be that we cannot prove that $t_{i} \downarrow$ until we know that certain of its subterms $s_{1}, \ldots, s_{n}$ are defined and satisfy

$$
\left[s_{1} \prec x \supset P\left(s_{1}\right)\right] \wedge \cdots \wedge\left[\left(s_{n} \prec x \supset P\left(s_{n}\right)\right],\right.
$$

and so on. Indeed, as we shall see, that is necessary to establish closure under nested recursion on the $\prec$ ordering. This leads in the next section to the precise statement of BR in the language of FA augmented by a new function symbol.

4.1. Defining $\mathcal{U}_{0}(\mathrm{FA}+\mathrm{BR})$. In the formulation of the rules below we use a binary relation $\prec$ whose characteristic function is given by a closed term $t_{\prec}$ for which $\mathcal{U}_{0}$ (FA) proves $t_{\prec}: \mathrm{N}^{2} \rightarrow\{0,1\}$. We write $x \prec y$ instead of $t_{\prec} x y=0$ and further assume that $\prec$ is a linear ordering with least element 0 , provably in $\mathcal{U}_{0}(\mathrm{FA})$.

In the following let $f$ denote a new constant of our applicative language. There are no nonlogical axioms for $\mathrm{f}$; it serves as an arbitrary (or anonymous) function from $\mathrm{N}$ to $\mathrm{N}$, representing a possibly infinite descending sequence along a given ordering.

The rule NDS $(f, \prec)$ says that for each possibly infinite descending chain $f$ w.r.t. $\prec$ there is an $x$ such that $\mathrm{f} x=0$. Formally, it is given as follows:

$$
\begin{gathered}
u \in \mathrm{N} \rightarrow \mathrm{f} u \in \mathrm{N}, \\
u \in \mathrm{N}, \mathrm{f} u \neq 0 \rightarrow \mathrm{f}\left(u^{\prime}\right) \prec \mathrm{f} u, \\
u \in \mathrm{N}, \mathrm{f} u=0 \rightarrow \mathrm{f}\left(u^{\prime}\right)=0 \\
(\exists x \in \mathrm{N})(\mathrm{f} x=0)
\end{gathered}
$$

Next, the Bar Rule BR is spelled out in detail for the case of nesting level two and a predicate with one parameter. The general case for nesting of arbitrary level and number of parameters is analogous. 
Let $\overline{s^{r}}=s_{1}^{r}, \ldots, s_{n}^{r}$ and $\overline{s^{p}}=s_{1}^{p}, \ldots, s_{n}^{p}$ be sequences of terms of length $n$, and let $\overline{t^{r}}=t_{1}^{r}, \ldots, t_{m}^{r}$ and $\overline{t^{p}}=t_{1}^{p}, \ldots, t_{m}^{p}$ be sequences of terms of length $m$. The superscripts ' $\mathrm{r}$ ' and ' $\mathrm{p}$ ' stand for recursion and parameter, respectively.

The Bar Rule BR now reads as follows. Whenever we have derived the four premises

(1) $\operatorname{NDS}(f, \prec)$

(2) $x, y \in \mathrm{N} \rightarrow \overline{s^{r}} \in \mathrm{N} \wedge \bar{s}^{p} \in \mathrm{N}$

(3) $x, y \in \mathrm{N}, \bigwedge_{i}\left[s_{i}^{r} \prec x \supset P\left(s_{i}^{r}, s_{i}^{p}\right)\right] \rightarrow \bar{t}^{r} \in \mathrm{N} \wedge \overline{t p} \in \mathrm{N}$

(4) $x, y \in \mathrm{N}, \bigwedge_{i}\left[s_{i}^{r} \prec x \supset P\left(s_{i}^{r}, s_{i}^{p}\right)\right], \bigwedge_{j}\left[t_{j}^{r} \prec x \supset P\left(t_{j}^{r}, t_{j}^{p}\right)\right] \rightarrow P(x, y)$

we can infer $x, y \in \mathrm{N} \rightarrow P(x, y){ }^{14}$

The new unfolding system $\mathcal{U}_{0}(\mathrm{FA}+\mathrm{BR})$ is the extension of $\mathcal{U}_{0}(\mathrm{FA})$ by this rule.

4.2. Nested recursion and well-foundedness in $\mathcal{U}_{0}(\mathrm{FA}+\mathrm{BR})$. In order to show that Peano Arithmetic PA is a lower bound to the proof-theoretic strength of $\mathcal{U}_{0}(\mathrm{FA}+\mathrm{BR})$, it will be shown that whenever we have derived NDS $(f, \prec)$ in $\mathcal{U}_{0}(\mathrm{FA}+\mathrm{BR})$, for a specific ordering $\prec$, then we can use the Bar Rule BR in order to justify function definitions by nested recursion along $\prec$. To see this, let us look at the following example.

Let $G, H, k, l, m, p$ be previously defined functions on the natural numbers which are suitably represented by terms that are provably total in $\mathcal{U}_{0}(\mathrm{FA}+\mathrm{BR})$. Using the fixed point operator of our combinatory universe, we can define a new function $F$ by nested level 2 recursion and parameter substitution to satisfy the following partial equalities for $0 \neq x, y \in \mathrm{N}$ :

$$
\begin{aligned}
& F(0, y) \simeq H(y) \\
& F(x, y) \simeq G\left(x, y, F\left(k\left(x, y, F\left(l(x, y)_{x}, y\right)\right)_{x}, p\left(x, y, F\left(m(x, y)_{x}, y\right)\right)\right)\right)
\end{aligned}
$$

where as usual $(r)_{x}$ is $r$ if $r \prec x$ and 0 otherwise. We need to show that $\mathcal{U}_{0}(\mathrm{FA}+\mathrm{BR})$ proves the implication $x, y \in \mathrm{N} \rightarrow F(x, y) \in \mathrm{N}$ and want to achieve this by substituting $P(x, y)$ by $(F(x, y) \in \mathrm{N})$ in the Bar Rule BR. Further, we have to specify the term sequences for the premises of BR. We set $n=2$ and $m=1$ and choose the following terms:

$$
\begin{aligned}
s_{1}^{r} & =l(x, y)_{x}, & s_{1}^{p} & =y \\
s_{2}^{r} & =m(x, y)_{x}, & s_{2}^{p} & =y \\
t_{1}^{r} & =k\left(x, y, F\left(l(x, y)_{x}, y\right)\right)_{x}, & t_{1}^{p} & =p\left(x, y, F\left(m(x, y)_{x}, y\right)\right) .
\end{aligned}
$$

It is now easy to see that the two premises (2) and (3) of BR are derivable using this choice and, hence, together with NDS( $\mathrm{f}, \prec)$, we obtain $x, y \in \mathrm{N} \rightarrow F(x, y) \in \mathrm{N}$ as desired.

This example is typical and leads us to

THEOREM 4.1. Assume that NDS(f, $\prec)$ is derivable in the system $\mathcal{U}_{0}(\mathrm{FA}+\mathrm{BR})$. Then $\mathcal{U}_{0}(\mathrm{FA}+\mathrm{BR})$ justifies nested recursion along $\prec$.

In the following let us assume that for each ordinal $\alpha<\varepsilon_{0}$ we have a standard primitive recursive well-ordering $\prec_{\alpha}$ of ordertype $\alpha$. Further, let us write NDS(f, $\left.\alpha\right)$ for NDS(f, $\left.\prec_{\alpha}\right)$.

${ }^{14}$ In the formulation of this rule, we have used the shorthand $r \prec x \supset A$ for the formula $t \prec r x=$ $1 \vee A$. 
The crucial ingredient of the argument to show that $\mathcal{U}_{0}(\mathrm{FA}+\mathrm{BR})$ derives NDS $(\mathrm{f}, \alpha)$ for each $\alpha<\varepsilon_{0}$ is the previously mentioned result by Tait (1961) that nested recursion on $\omega \alpha$ entails ordinary recursion on $\omega^{\alpha}$ or, more useful in our setting, nested recursion on $\omega \alpha$ entails NDS $\left(f, \omega^{\alpha}\right)$. A very concrete and compact presentation of the argument in Tait (1961) has recently been given by Tait (2006b) in a personal communication with the first author. That is presented in the appendix to this paper. From there it will be seen that the argument can be directly formalized in $\mathcal{U}_{0}(\mathrm{FA}+\mathrm{BR})$; some more specific comments are to be found in the appendix. ${ }^{15}$

THEOREM 4.2. Provably in $\mathcal{U}_{0}(\mathrm{FA}+\mathrm{BR})$, nested recursion along $\omega \alpha$ entails $\mathrm{NDS}\left(\mathrm{f}, \omega^{\alpha}\right)$.

Clearly, $\mathcal{U}_{0}(\mathrm{FA}+\mathrm{BR})$ proves NDS(f, $\left.\omega 2\right)$ and hence we have nested recursion along $\omega 2$, which in turn entails NDS $\left(f, \omega^{2}\right)$; further, nested recursion on $\omega^{2}$ gives us NDS $\left(\mathfrak{f}, \omega^{\omega}\right)$ and thus nested recursion along $\omega^{\omega}=\omega\left(\omega^{\omega}\right)$. Then we can derive NDS $\left(\mathbf{f}, \omega^{\omega^{\omega}}\right)$ and so on.

The upshot is that $\mathcal{U}_{0}(\mathrm{FA}+\mathrm{BR})$ derives $\operatorname{NDS}\left(\mathrm{f}, \omega_{n}\right)$ for each natural number $n$, where as usual we set $\omega_{0}=\omega$ and $\omega_{n+1}=\omega^{\omega_{n}}$. This gives us the crucial result of this section.

COROLLARY 4.3. We have for each $\alpha<\varepsilon_{0}$ that $\mathcal{U}_{0}(\mathrm{FA}+\mathrm{BR})$ derives $\mathrm{NDS}(\mathrm{f}, \alpha)$.

In the next subsection we will see that this lower bound is indeed sharp.

4.3. $\mathcal{U}_{0}(\mathrm{FA}+\mathrm{BR})$ does not go beyond $\mathrm{PA}$. In the following we will sketch an embedding of $\mathcal{U}_{0}(\mathrm{FA}+\mathrm{BR})$ in a version of $\mathrm{ACA}_{0}$, the well-known subsystem of second order arithmetic based on arithmetical comprehension together with set induction. For that purpose, let $\mathcal{L}_{2}$ denote the usual language of second order arithmetic extending the first order language $\mathcal{L}_{1}$ by set variables $X, Y, Z, \ldots$ and function variables $f, g, h, \ldots$ We assume that $\mathcal{L}_{2}$ also contains our free relation symbols $\bar{P}$. The formulas of $\mathcal{L}_{2}$ are defined in the usual way. A formula is called arithmetic, if it does not contain bound set or function variables; it may contain, however, free set and function variables as well as positive and negative occurrences of the relations $\bar{P}$.

$\mathrm{ACA}_{0}$ is the $\mathcal{L}_{2}$ theory comprising the axioms of Peano Arithmetic PA, for each arithmetic formula $A(x)$ the comprehension axiom

$$
(\exists X)(\forall x)(x \in X \leftrightarrow A(x)),
$$

the graph principle relating functions and sets, ${ }^{16}$

$$
(\forall x)(\exists ! y)\langle x, y\rangle \in X \rightarrow(\exists f)(\forall x)\langle x, f(x)\rangle \in X,
$$

and, finally, induction on the natural numbers for sets:

$$
0 \in X \wedge(\forall x)\left(x \in X \rightarrow x^{\prime} \in X\right) \rightarrow(\forall x)(x \in X) .
$$

It is well known that $\mathrm{ACA}_{0}$ is a conservative extension of Peano Arithmetic PA.

15 In Tait (2006a), Sec. 6 Tait gives an analysis of a proposed proof of Bernays in Hilbert \& Bernays (1970), pp. 533-535 of induction up to $\varepsilon_{0}$. Tait's critical inspection of Bernays' argument reveals that the result indeed only shows that NDS(f, $\left.\omega^{\alpha}\right)$ can be reduced to nested recursion on $\omega \alpha$. Bernays' attractive construction is spelled out in detail in Tait (2006a). In contrast to Tait's own argument in Tait $(1961,2006 \mathrm{~b})$ which uses the definition by nested recursion of a type one function, in Bernays' proof one defines a type two functional by nested recursion. Due to the logical restrictions of our unfolding system for finitist arithmetic, Tait's original argument is more suitable for our formalization and hence we will follow Tait $(1961,2006 \mathrm{~b})$ in the sequel.

16 Below, $\langle\cdot, \cdot\rangle$ denotes a primitive recursive pairing function. 
Let us now describe the interpretation of $\mathcal{U}_{0}(\mathrm{FA}+\mathrm{BR})$ in $\mathrm{ACA}_{0}$. It is a modification of the translation of $\mathcal{U}_{0}(\mathrm{FA})$ in PRA, but now taking into account the function constant $f$ in the language of $\mathcal{U}_{0}(\mathrm{FA}+\mathrm{BR})$. For that purpose, we fix a function variable $f$ in $\mathcal{L}_{2}$ and now translate $(a \cdot b)$ as $\{a\}^{f}(b)$, where $\{n\}^{f}$ for $n=0,1,2, \ldots$ is a standard enumeration of the functions that are partial recursive in $f$. Accordingly, $\mathrm{f}$ is interpreted as a natural number $i$ for which $\{i\}^{f}(x) \simeq f(x)$.

This determines for each formula $A$ its translation $A^{*}$. Observe that $A^{*}$ is an arithmetic formula of $\mathcal{L}_{2}$ containing the function parameter $f$. The $*$ translation is extended to sequents and rules of inference as expected.

Clearly, the $*$ translation of all axioms of $\mathcal{U}_{0}(\mathrm{FA}+\mathrm{BR})$ and the induction rule of FA are derivable in $\mathrm{ACA}_{0}$. Moreover, the treatment of the substitution rule (Subst') is analogous to $\mathcal{U}_{0}(\mathrm{FA})$ by using the fact that the axioms of $\mathrm{ACA}_{0}$ are closed under substitutions of the free relations $\bar{P}$ by arithmetic formulas.

LEMMA 4.4. If $A_{1}, A_{2}, \ldots, A_{n} \Rightarrow A$ is a derivable rule of $\mathrm{ACA}_{0}$ and $\bar{B}$ are arithmetic formulas of $\mathcal{L}_{2}$, then also $A_{1}[\bar{B} / \bar{P}], A_{2}[\bar{B} / \bar{P}], \ldots, A_{n}[\bar{B} / \bar{P}] \Rightarrow A[\bar{B} / \bar{P}]$ is a derivable rule of $\mathrm{ACA}_{0}$.

The crucial step of the embedding, of course, is the treatment of the Bar Rule BR.

LEMMA 4.5. The * translation of the Bar Rule $\mathrm{BR}$ is an admissible rule of $\mathrm{ACA}_{0}$.

Proof. Let us assume that the $*$ translation of the rule NDS $(f, \prec)$ is derivable in $\mathrm{ACA}_{0} \cdot{ }^{17}$ Thus we can conclude that the following is derivable in $\mathrm{ACA}_{0}$ :

$$
(\forall x)\left[\left(f(x) \neq 0 \rightarrow f\left(x^{\prime}\right) \prec f(x)\right) \wedge\left(f(x)=0 \rightarrow f\left(x^{\prime}\right)=0\right)\right] \rightarrow(\exists x)(f(x)=0) .
$$

This entails that $\prec$ is a well-founded relation, which in turn is equivalent in $\mathrm{ACA}_{0}$ to the principle of transfinite induction for all sets, $(\forall X) \operatorname{TI}(\prec, X)$, where $\operatorname{TI}(\prec, X)$ stands for

$$
(\forall x)[(\forall y \prec x)(y \in X) \rightarrow(x \in X)] \rightarrow(\forall x)(x \in X) .
$$

But trivially, $(\forall X) \operatorname{TI}(\prec, X)$ entails $\operatorname{TI}(\prec, A)$ for each arithmetic formula $A$, that is, we have transfinite induction along our ordering $\prec$ in $\mathrm{ACA}_{0}$ for every arithmetic property $A$.

It is now readily seen that our Bar Rule BR is valid by applying transfinite induction to the predicate $(\forall y) P(x, y)$.

We can now summarize our findings in the following main theorem.

THEOREM 4.6. $\mathcal{U}_{0}(\mathrm{FA}+\mathrm{BR})$ is contained in $\mathrm{ACA}_{0}$ via the translation $*$.

COROLlary 4.7. $\mathcal{U}_{0}(\mathrm{FA}+\mathrm{BR})$ is proof-theoretically equivalent to Peano Arithmetic PA.

In particular, we have that provably total terms of type $\left(\mathrm{N}^{n} \rightarrow \mathrm{N}\right)$ give rise to $<\varepsilon_{0^{-}}$ recursive functions.

COROLLARY 4.8. Let $t$ be a closed $\mathcal{U}_{0}(\mathrm{FA})$ term and assume that $\mathcal{U}_{0}(\mathrm{FA}+\mathrm{BR})$ proves the sequent $\bar{u} \in \mathrm{N} \rightarrow t(\bar{u}) \in \mathrm{N}$. Then $t$ defines $a<\varepsilon_{0}$-recursive function.

17 We can assume that the function variable $f$, which gives the interpretation for the function constant $f$, only occurs as a parameter in this derivation, since we are only interested in derivations coming from $\mathcal{U}_{0}(\mathrm{FA}+\mathrm{BR})$, where we simply have $\mathrm{f}$ as a constant. 
4.4. $\mathcal{U}(\mathrm{FA}+\mathrm{BR})$ is contained in $\mathrm{ACA}_{0}$. The full unfolding system with Bar Rule, $\mathcal{U}(\mathrm{FA}+\mathrm{BR})$, does not go beyond Peano Arithmetic in strength. For, we can take the preceding translation of $\mathcal{U}_{0}(\mathrm{FA}+\mathrm{BR})$ in $\mathrm{ACA}_{0}$ and extend it by the interpretation of the predicate part of $\mathcal{U}(F A+B R)$ in literally the same way as in Section 3.5. This immediately yields the desired embedding of $\mathcal{U}(\mathrm{FA}+\mathrm{BR})$ in $\mathrm{ACA}_{0}$.

THEOREM 4.9. $\mathcal{U}_{0}(\mathrm{FA}+\mathrm{BR}), \mathcal{U}_{1}(\mathrm{FA}+\mathrm{BR})$, and $\mathcal{U}(\mathrm{FA}+\mathrm{BR})$ are all proof-theoretically equivalent to Peano Arithmetic PA.

COROLLARY 4.10. Let $t$ be a closed $\mathcal{U}_{0}(\mathrm{FA})$ term and assume that $\mathcal{U}(\mathrm{FA}+\mathrm{BR})$ proves the sequent $\bar{u} \in \mathrm{N} \rightarrow t(\bar{u}) \in \mathrm{N}$. Then $t$ defines $a<\varepsilon_{0}$-recursive function.

A Well-foundedness of exponentiation. In this appendix we will present Tait's argument showing that nested recursion on $\omega \delta$ entails ordinary recursion on $\omega^{\delta}$ or, as we will use it in our context, nested recursion on $\omega \delta$ entails NDS $\left(\mathrm{f}, \omega^{\delta}\right)$. We will follow the presentation of this argument in Tait (2006b), which is essentially the same as in Tait (1961), p. 248 ff., but more compact and readable since it refers directly to the usual ordering of type $\varepsilon_{0}$, whereas Tait (1961) uses a more general notion of standard ordering. We will see that the argument can be directly formalized in $\mathcal{U}_{0}(\mathrm{FA}+\mathrm{BR})$.

In the following we will work with (codes of) ordinals below $\varepsilon_{0}$ and assume that $<$ denotes the corresponding ordering relation on them. Further, let $f$ be a fixed function from $\omega$ to $\omega^{\delta}$ satisfying for all natural numbers $n$ the condition

$$
f(n)>0 \rightarrow f(n+1)<f(n) \quad \text { and } \quad f(n)=0 \rightarrow f(n+1)=0 .
$$

Given an ordinal $\alpha<\omega^{\delta}$ in its normal form

$$
\alpha=\omega^{\alpha_{1}} a_{1}+\cdots+\omega^{\alpha_{n}} a_{n}
$$

where $\delta>\alpha_{1}>\cdots>\alpha_{n}$ and $a_{i}<\omega(1 \leq i \leq n)$, we set

$$
\begin{aligned}
& \alpha\{i\}:=\omega^{\alpha_{1}} a_{1}+\cdots+\omega^{\alpha_{n}} a_{k} \quad(k=\min (n, i)) \\
& \alpha[i]:= \begin{cases}\omega \alpha_{i}+a_{i} & \text { if } 0<i \leq n \\
0 & \text { if } n<i .\end{cases}
\end{aligned}
$$

Clearly, $\alpha[i]<\omega \delta$ and $0\{i\}=0[i]=0$. The following key lemma relates these notions.

LEMMA A.1. We have that $\alpha\{i+1\}<\beta\{i+1\}$ if and only if

$$
\alpha\{i\}<\beta\{i\} \vee(\alpha\{i\}=\beta\{i\} \wedge \alpha[i+1]<\beta[i+1]) .
$$

The crucial step in Tait's argument is to define a function $\mu: \omega^{2} \rightarrow \omega$ such that (writing $\mu_{i}(j)$ for $\left.\mu(i, j)\right)$,

$$
f\left(j+\mu_{i}(j)\right)=0 \vee f\left(j+\mu_{i}(j)\right)\{i\}<f(j)\{i\} .
$$

It will then suffice to choose $\mu_{0}(0)$ as a root of $f$, since according to $(\star \star)$, we have that $f\left(\mu_{0}(0)\right)=0$.

The definition of $\mu_{i}(j)$ will be by nested recursion on $f(j)[i+1]<\omega \delta$. We first describe the definition of $\mu_{i}(j)$ informally and give the explicit definition by nested recursion afterwards.

Let $n$ be the number of summands in the normal form of $f(j)$. If $i \geq n$, we may simply set $\mu_{i}(j)=1$; then $(\star \star)$ holds due to property $(\star)$ of our given function $f$. So assume 
$0 \leq i<n$. Because $f(j)[i+2]<f(j)[i+1]$, we can use $\mu_{i+1}(j)=\bar{\mu}$ in the definition of $\mu_{i}(j)$. Hence, according to ( $\left.\star \star\right)$ we have for $\bar{\mu}$ that either (A 1) or (A 2) holds:

$$
\begin{gathered}
f(j+\bar{\mu})=0 \\
f(j+\bar{\mu})\{i+1\}<f(j)\{i+1\} .
\end{gathered}
$$

If (1) holds, we set $\mu_{i}(j)=\bar{\mu}$. In case of (A 2), we use the lemma above to obtain one of the following properties (A 3) or (A 4):

$$
\begin{gathered}
f(j+\bar{\mu})\{i\}<f(j)\{i\} \\
f(j+\bar{\mu})\{i\}=f(j)\{i\} \wedge f(j+\bar{\mu})[i+1]<f(j)[i+1] .
\end{gathered}
$$

In case of (A 3), we again set $\mu_{i}(j)=\bar{\mu}$. If (A 4) holds, then clearly $\mu_{i}(j+\bar{\mu})=\overline{\bar{\mu}}$ is defined. In this case we set $\mu_{i}(j)=\bar{\mu}+\overline{\bar{\mu}}$. Then we can verify, using property $(\star \star)$ for $\overline{\bar{\mu}}$, that one of the following conditions (A 5) or (A 6) holds:

$$
\begin{gathered}
f\left(j+\mu_{i}(j)\right)=f((j+\bar{\mu})+\overline{\bar{\mu}})=0 \\
f\left(j+\mu_{i}(j)\right)\{i\}<f(j+\bar{\mu})\{i\}=f(j)\{i\} .
\end{gathered}
$$

This is as desired and concludes the definition of the function $\mu_{i}(j)$. Summarizing, $\mu_{i}(j)$ has been defined to satisfy the following equation:

$$
\mu_{i}(j)=\left\{\begin{array}{l}
1, \quad \text { if } i \geq n \\
\mu_{i+1}(j), \quad \text { if } f\left(j+\mu_{i+1}(j)\right)=0 \text { or } f\left(j+\mu_{i+1}(j)\right)\{i\}<f(j)\{i\} \\
\mu_{i+1}(j)+\mu_{i}\left(j+\mu_{i+1}(j)\right), \quad \text { else. }
\end{array}\right.
$$

It remains to explicitly express the definition of $\mu_{i}(j)$ by nested recursion on $\omega \delta$. For that purpose let the function $m_{i}(\alpha, j)$ be defined by nested recursion along $\alpha<\omega \delta$ as follows:

$$
m_{i}(\alpha, j)=\left\{\begin{array}{l}
1, \quad \text { if } \alpha=0 \\
\widetilde{m}, \quad \text { if } f(j+\widetilde{m})=0 \text { or } f(j+\widetilde{m})\{i\}<f(j)\{i\} \\
\widetilde{m}+m_{i}\left((f(j+\widetilde{m})[i+1])_{\alpha}, j+\widetilde{m}\right), \quad \text { else. }
\end{array}\right.
$$

where $\widetilde{m}$ is an abbreviation for the term $m_{i+1}\left((f(j)[i+2])_{\alpha}, j\right)$. Clearly, this is a valid nested recursion and we readily see that indeed

$$
\mu_{i}(j)=m_{i}(f(j)[i+1], j) .
$$

To conclude, let us briefly address the formalization of Tait's argument in the system $\mathcal{U}_{0}(\mathrm{FA}+\mathrm{BR})$. Assuming that the NDS property holds for the standard ordering of type $\omega \delta$, we can define the function $m_{i}(\alpha, j)$ by nested recursion along $\omega \delta$ as we have indicated in Section 4. This nested recursion uses the function constant $f$ (representing a possibly infinite descending sequence in $\left.\omega^{\delta}\right)$ as a parameter. The totality of $m_{i}\left(\right.$ resp. $\left.\mu_{i}\right)$ and all necessary properties (e.g., ( $\star \star))$ can then be derived by means of BR. 


\section{BIBLIOGRAPHY}

Beeson, M. J. (1985). Foundations of Constructive Mathematics: Metamathematical Studies. Berlin, Germany: Springer.

Feferman, S. (1975). A language and axioms for explicit mathematics. In Crossley, J., editor. Algebra and Logic, Volume 450 of Lecture Notes in Mathematics. Berlin, Germany: Springer, pp. 87-139.

Feferman, S. (1996). Gödel's program for new axioms: Why, where, how and what? In Hájek, P., editor. Gödel '96, Volume 6 of Lecture Notes in Logic. Berlin, Germany: Springer, pp. 3-22.

Feferman, S., Baaz, M., Papadimitriou, C. H., Putnam, H., Scott, D., \& Harper, C. L., Jr. Lieber Herr Bernays!, Lieber Herr Gödel!. Gödel on finitism, constructivity and Hilbert's program. In Baaz, M., et al., editor. Horizons of Truth: Logics, Foundations of Mathematics, and the Quest for Understanding the Nature of Knowledge. New York: Cambridge University Press. Reprinted in Dialectica 62 (2008), 179-203.

Feferman, S., \& Strahm, T. (2000). The unfolding of non-finitist arithmetic. Annals of Pure and Applied Logic, 104(1-3), 75-96.

Feferman, S., \& Strahm, T. (2001). Unfolding finitist arithmetic (Abstract). Bulletin of Symbolic Logic, 7(1), 111-112.

Gödel, K. (1931). Über formal unentscheidbare Sätze der Principia Mathematica und verwandter Systeme. Monatshefte für Mathematik und Physik, 38, 173-198.

Gödel, K. (1995). In Feferman, S., Dawson, J. W., Jr., Goldfarb, W., Parsons, C., \& Solovay, R. N. Collected Works Vol. III. New York: Oxford University Press.

Gödel, K. (2003). In Feferman, S., Dawson, J. W., Jr., Goldfarb, W., Parsons, C., \& Sieg, W. Collected Works Vol. IV. Oxford: Clarendon Press.

Hilbert, D., \& Bernays, P. (1968). Grundlagen der Mathematik (2nd edition), Vol. I. Berlin, Germany: Springer.

Hilbert, D., \& Bernays, P. (1970). Grundlagen der Mathematik (2nd edition), Vol. II. Berlin, Germany: Springer.

Kreisel, G. (1960). Ordinal logics and the characterization of informal concepts of proof. In Todd, J. A., editor. Proceedings International Congress of Mathematicians, 14-21 August 1958. Cambridge: Cambridge University Press, pp. 289-299.

Kreisel, G. (1965). Mathematical logic. In Saaty, T. L., editor. Lectures on Modern Mathematics, Vol. 3. New York: Wiley, pp. 95-195.

Kreisel, G. (1970). Principles of proof and ordinals implicit in given concepts. In Kino, A., Myhill, J., and Vesley, R. E., editors. Intuitionism and Proof Theory. Amsterdam, The Netherlands: North Holland, pp. 489-516.

Mints, G. (1973). Quantifier-free and one-quantifier systems. Journal of Soviet Mathematics, 1, 71-84.

Parsons, C. (1970). On a number theoretic choice schema and its relation to induction. In Myhill, J., Kino, A., and Vesley, R., editors. Intuitionism and Proof Theory, Proceedings of the Summer Conference at Buffalo N.Y., 1968. Amsterdam, The Netherlands: North Holland, pp. 459-473.

Parsons, C. (1998). Finitism and intuitive knowledge. In Schirn, M., editor. The Philosophy of Mathematics Today. Oxford: Oxford University Press, pp. 249-270.

Sieg, W. (1985). Fragments of arithmetic. Annals of Pure and Applied Logic, 28, 33-71.

Strahm, T. (2000). The non-constructive $\mu$ operator, fixed point theories with ordinals, and the bar rule. Annals of Pure and Applied Logic, 104(1-3), 305-324.

Tait, W. (1961). Nested recursion. Mathematische Annalen, 143, 236-250. 
Tait, W. (1968). Constructive reasoning. In van Rootselaar, B., and Staal, J. F., editors. Logic, Methodology and Philosophy of Science III. Amsterdam, The Netherlands: North Holland, pp. 185-199.

Tait, W. (1981). Finitism. Journal of Philosophy, 78, 524-546.

Tait, W. (2005). The Provenance of Pure Reason. New York: Oxford University Press.

Tait, W. (2006a). Gödel's correspondence on proof theory and constructive mathematics. Philosophia Mathematica (III), 14, 76-111.

Tait, W. (2006b). Wellfoundedness of exponentiation. Personal communication.

Troelstra, A., \& van Dalen, D. (1988). Constructivism in Mathematics, Vol. I. Amsterdam, The Netherlands: North-Holland.

Zach, R. (2003). Hilbert's Program. Stanford Encyclopedia of Philosophy. Available from: http://plato.stanford.edu/entries/hilbert-program/.

Zach, R. (2006). Hilbert's program then and now. In Jacquette, D., editor. Philosophy of Logic, Volume 5 of Handbook of the Philosophy of Science. Amsterdam, The Netherlands: Elsevier, pp. 411-447.

\author{
DEPARTMENT OF MATHEMATICS \\ STANFORD UNIVERSITY \\ STANFORD, CA 94305 \\ E-mail: feferman@stanford.edu
INSTITUT FÜR INFORMATIK UND ANGEWANDTE MATHEMATIK
UNIVERSITÄT BERN
NEUBRÜCKSTRASSE 10
CH-3012 BERN, SWIZTERLAND \\ E-mail: strahm@iam.unibe.ch
}

